\title{
THE NUMERICAL RESULTS OF DIVERSE SYSTEMS OF BREEDING ${ }^{1}$
}

\author{
H. S. JENNINGS \\ Johns Hopkins University, Baltimore, Maryland
}

[Received August 26, 1915]

CONTENTS

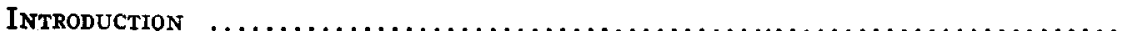

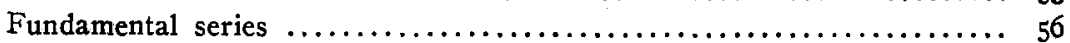

The rate of change in the diverse systems of breeding............. 58

Derivation of the formulæ................................ 6i

Method of presentation; directions for the use of the formulæ....... 62

I. TyPicAL FACTORS, NOT SEX-LINKED................................. 64

a. Random mating .......................................... 64

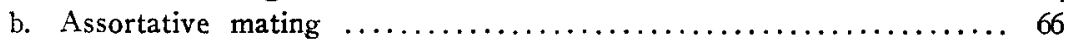

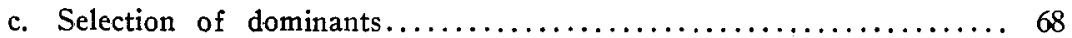

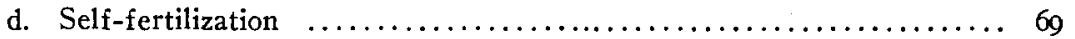

Inbreeding $\ldots \ldots \ldots \ldots \ldots \ldots \ldots \ldots \ldots \ldots \ldots \ldots \ldots \ldots \ldots \ldots, 7_{0}$

e. Brother $\times$ sister mating $\ldots \ldots \ldots \ldots \ldots \ldots \ldots \ldots \ldots \ldots \ldots \ldots, 7_{\mathrm{I}}$

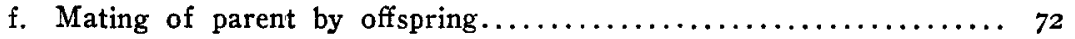

i. Same father successively bred $\ldots \ldots \ldots \ldots \ldots \ldots \ldots \ldots \ldots, 73$

ii. Parent by offspring, alternating system.............. 74

iii. Half the progeny bred to one parent, half to the other..... 77

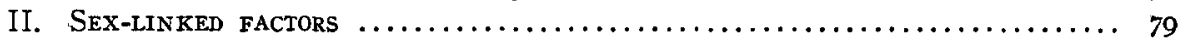

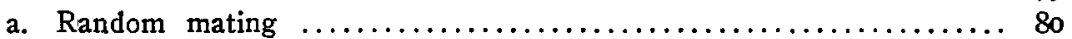

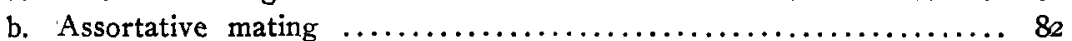

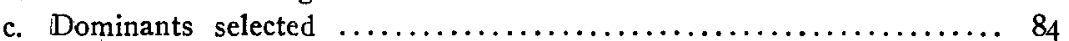

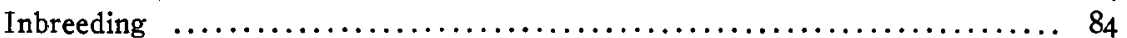

d. Mating of brother and sister............................... 84

e. Mating of parent by off spring $\ldots \ldots \ldots \ldots \ldots \ldots \ldots \ldots \ldots \ldots \ldots, 86$

i. Same father successively bred..................... 86

ii. All females bred to father, all males to mother........... 86

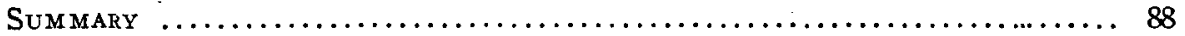

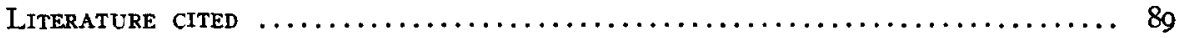

\section{INTRODUCTION}

To work out without formulae the results of almost any system of breeding long continued is a most laborious and time-consuming task, while a formula serves as a machine; one puts in the data and grinds out the results without labor. The present paper is a collection of formulae for the results of most of the ustual systems of breeding. The systems dealt with are: random mating, assortative mating of domi-

${ }^{1}$ From the Zoölogical Laboratory of the Johns Hopkins University. 
nants with dominants, recessives with recessives; selection of dominants alone; self-fertilization; and the various systems of inbreeding (of which a considerable variety exists). The present formulae give the distribution of single characters, sex-linked or not sex-linked, in continued breeding by such systems. A later paper will deal similarly with the cases where there are two or more characters, independent or linked together in various degrees. Most of the formulae are here presented for the first time; the few previously worked out are included in order to make as complete and systematic a collection as possible.

Strict Mendelian heredity presents a rigid scheme; its results can be computed; it is a problem in arithmetic. To work out its numerical formulae does not involve taking ground on the question whether all inheritance actually is of this rigid character. To discover whether the results of inheritance are continually subject to evolutionary change, as some hold, it is necessary to know first what would be the results without such evolutionary change. The results of actual experimentation may then be compared with what the formulae demand, in order to determine whether the processes of nature are or are not equally rigid.

The type of the formulae here set forth is the well-known I:2:I ratio observed in the second filial generation when two individuals having such factors as $A A$ and $a a$ are crossed. Recent developments in the study of heredity have introduced some complications into the relations; sex-linked characters follow rules of their own, and linkage greatly modifies the rules of distribution before known. These taken with the various systems of breeding give results requiring a variety of formulae for their working out.

The formulae for the first and second filial generations after making a cross between two parents differing in one or a few factors are of course well known. Pearson (rgo4) shows that the formula $1 / 4 A A+$ $\mathrm{r} / 2 A a+\mathrm{r} / 4 a a$ holds for any generation for the progeny by random mating of $A A$ and $a a$, and HARDY (I908) has made the same point. The present writer (JENNINGS I9I2) gave a formula for the results of self-fertilization. PEARL (I914 a) worked out the results of inbreeding of brother and sister among the progeny of $A A \times a a$, and gave a formula (PEARL I9I4 b) showing how the constitution of any generation can be derived when that of the preceding generation is known. I gave a general formula for the proportion of homozygotes in any generation in this case (JENNINGS I9I4), a formula superseded by the simpler ones in the present paper. FISH (I9r4) has studied the results of inbreeding, both of parent by offspring and of brother by sister; he gives the per- 


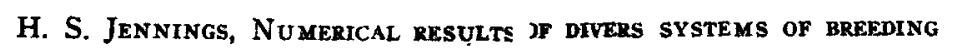

TABLE 1. Fundamental series in Mexdelian breeding.

How formed

\begin{tabular}{|c|c|c|c|c|c|c|c|c|c|c|c|c|c|c|c|c|c|c|c|c|c|c|c|}
\hline$n$ & $x+1$ & 0 & $\mathbf{x}$ & 2 & 3 & 4 & 5 & 6 & 7 & 8 & 9 & ro & II & 12 & 13 & 14 & 15 & 16 & 17 & 18 & 19 & 20 & Formula \\
\hline $\mathbf{B}$ & $2 \mathrm{x}$ & $I$ & 2 & 4 & 8 & 16 & 32 & 64 & 128 & 256 & 512 & 1024 & 2048 & 4096 & 8192 & 384 & 368 & 5536 & 1072 & 21. & 524288 & 0 & $2^{n}$ \\
\hline c & $2 x+\mathbf{I}$ & 0 & I & 3 & 7 & 15 & $3^{1}$ & 63 & 127 & 255 & 51 & 10 & a & 40 & 8191 & 6383 & 270 & 0553 & 1071 & 02143 & 524287 & 20 & -1 \\
\hline & $2 x-I$ & & 2 & 3 & 5 & 9 & 17 & 33 & 65 & 129 & 257 & 5 & 0. & $\infty$ & 4097 & 8193 & 6385 & 32 & 65 & 131073 & 262 & 52 & $2^{n-1}+1$ \\
\hline I & $2 x+1$ & & & 2 & 5 & II & 23 & 47 & 95 & I9I & 383 & $76 \%$ & I533 & $309 x$ & $6 \times 43$ & 2287 & 24575 & 49151 & 98303 & 196607 & 393215 & 786431 & $2^{n-1}+2^{n-2}-1$ \\
\hline & Sum of two preceding & 0 & $\mathbf{I}$ & $\mathbf{I}$ & 2 & 3 & 5 & 8 & 13 & 21 & 34 & 55 & 8 & 144 & 233 & 377 & 61 & . & 1597 & 288. & $\mathbf{I}$ & 6765 & \\
\hline G & $2 x+1$, then $2 x-1$ & 0 & $I$ & I & 3 & 5 & II & $2 \mathrm{I}$ & 43 & 85 & 171 & 341 & 683 & 1365 & 2731 & $546 \mathrm{r}$ & 0923 & 21845 & $4369 r$ & 87381 & 174763 & 349525 & $\left(=\mathbf{B}_{n-1}-\mathbf{G}_{n-1}\right)$ \\
\hline & $\mathbf{G}-\mathbf{F}$ & o & $\mathbf{o}$ & $\mathbf{0}$ & I & 2 & 6 & 13 & 30 & 64 & 137 & 28 & 594 & 122 & 2498 & 5084 & 10313 & 2085 & 42 & 84: & $I 7$ & & \\
\hline I & $\mathbf{B}-\mathbf{G}-\mathbf{F}$ & $\mathbf{I}$ & o & 2 & 3 & 8 & 16 & 35 & .72 & 150 & 307 & 628 & 1276 & 2987 & 3228 & 10546 & 21 & 42704 & 85784 & 9 & 345344 & 692286 & $\left(=G_{n-1}-F_{n}\right)$ \\
\hline $\mathbf{J}$ & $\mathbf{B}_{n}-\mathbf{F}_{n+1}$ & $\circ$ & $\mathbf{I}$ & 2 & 5 & II & 24. & $5 \mathbf{I}$ & 107 & 222 & $457^{\circ}$ & 935 & 1904 & 363 & 7715 & 15774 & $3178 \mathrm{I}$ & 63939 & 128468 & 257963 & & 1037630 & \\
\hline $\mathrm{K}$ & $F_{n+2}$ & ० & 0 & I & 3 & 8 & 19 & 43 & 94 & 2 & 423 & 880 & 1875 & $36 \times 9$ & 7582 & 15397 & 31171 & 62952 & I2689 & 255379 & 513342 & 1030865 & $\left(=\mathrm{J}_{n}-\mathrm{F}_{n}\right)$ \\
\hline L & $B_{n}-F_{n+1}-G_{n-1}$ & & 2 & 2 & 6 & II & 24 & 48 & 99 & 200 & 406 & 819 & 1652 & 3324 & 6683 & 13420 & 26930 & 54003 & 108240 & 216856 & & 869632 & \\
\hline$M$ & ${ }_{3} B_{n}-F_{n+2}$ & 2 & 4 & 9 & 19 & 40 & 83 & $17 \pi$ & 350 & 713 & 1447 & 2928 & 5911 & frgil & 23966 & 48165 & 96707 & 194024 & 389035 & 779667 & I561918 & 3128017 & $\left(=B_{n+1}-K_{n}\right)$ \\
\hline
\end{tabular}

Genetics 1: Ja 1916 
centages of homozygotes in the latter case up to 25 inbreedings. $^{2}$ Other work of this sort is not known to me.

The questions which the formulae are designed to answer may be illustrated as follows: Suppose that one parent has the factors $A A$, the other $a a$; and that after their crossing there is assortative mating (dominants with dominants, recessives with recessives) for seven generations. What will then be the constitution of the population; what proportion will be $A A$, what $a a$, what $A a$ ? Further, if such mating is indefinitely continued, what are the limits to which the proportions of each of the three classes may finally attain? And how many generations must such breeding be continued before the limiting proportions are, for practical purposes, attained? Suppose that the parents differ, not in one pair of factors alone, but in several, how are these questions to be answered for each pair separately, and for the various possible combinations of them?

It should be emphasized of course that the results set forth in the formulae here given, hold with some degree of precision only if the experiment is carried out on an extensive scale, with many lines of descent. When the breeding is carried out with few lines of descent, the results given by the formulae are merely the most probable results.

We shall deal in part I with a single pair of typical Mendelian factors; in part II with single sex-linked factors.

We shall represent any single pair of alternative factors by $A$ and $a$, in the three possible combinations $A A, A a$ and $a a$.

It is clear that the outcome of any system of breeding depends not only on the system followed, but also on the constitution of the parents at the beginning. For many (not all) types of breeding the beginning is a cross between two parents. The matings of two parents may be $A A$ $\times A A ; a a \times a a ; A A \times a a ; A A \times A a ; A a \times A a$, or $A a \times a a$. The first two matings require no consideration, since by any system of breeding all the progeny are like the parents. The most important differing parental types are $A A \times a a$ and $A A \times A a$; these give diverse results, while from other crosses the results are similar to one or the other of these. We shall specify in each case both the system of breeding followed and the constitution of the parents.

When breeding is continued according to a given system for many generations, several types of results may be distinguished, so far as concerns the constitution of the population, and the formulae expressing it:

${ }^{2}$ FISH states, by what is evidently a slip, that the percentages are of heterozygotism.

Genetics 1: Ja 1916 
(a) In some cases the proportions (and formula) remain the same for all generations. It is mainly in random mating that this is the case. Examples are found in sections (I), (4), (37), etc. ${ }^{3}$

(b) The constitution of the population changes from generation to generation. In such cases it is often possible to give a simple formula based upon $n$, the number of generations; from it the result for any generation can be directly computed. Examples are (I3), (I6), (I7), etc.

(c) In other cases the proportions of the population give a series of values, not easily expressible as simple functions of $n$ alone. These cases are the most numerous and most interesting; they will be dealt with further presently.

(d) In some cases a general formula for any generation taken by itself is not obtained; the best that we can do is to give a formula for determining the constitution of any generation when that of the immediately preceding generation is known. Only a few cases of this sort have been found among those here dealt with, for example, (8), (53), (54), (55), etc.

\section{FUNDAMENTAL SERIES}

As just mentioned ((c), above), in most cases the population resulting from a given system of breeding shows in the successive generations a series of definite proportions for each of the possible combinations of factors present. The diverse series so obtained, whether from random mating, assortative mating, self-fertilization, or one of the various types of inbreeding, are almost all examples of certain simply derived mathematical series, or of their combinations. It will greatly facilitate the presentation of results and avoid much repetition, if we deal first in a general way with these simple series, giving their laws of formation, and presenting in a table the first twenty terms of each. Our later results may then be presented by designating the terms of the particular series which they give. These fundamental series are shown in table $\mathrm{I}$.

Aside from the series of natural numbers, designated $n$ in the table, the most fundamental series for Mendelian breeding is that of the successive powers of 2 ; this series is here designated B. Each term is derived from the preceding one by doubling it. The fractions expressing the proportions of the population having any particular combination of factors have as a rule terms of this series as denominators.

\footnotetext{
s The numbers in parentheses refer to the numbered sections or paragraphs in which the formula are given later, in the body of the paper.
} 
Closely related to the series of powers of 2 are several series (C, D and $\mathrm{E}$ in the table) in which any succeeding term is derived by doubling the preceding one, and adding or subtracting $\mathrm{I}$ to or from the result. Series $\mathrm{C}$ is formed by beginning with o, doubling each term and adding I to give the next term. Each term is I less than the corresponding term of B. Series D begins with 2 ; succeeding terms are obtained by doubling and subtracting $\mathrm{I}$. Each term is one more than the immediately preceding term of series $B$. (A series beginning with $I$ and formed by doubling each term and subtracting I plays a part in breeding, but as all its terms are $I$, it does not require elucidation.) Series $E$ is obtained by beginning with 2 , doubling and adding $\mathrm{I}$ to the preceding term. Each of its terms is the difference between a given term of $B$ and the preceding term of $D$.

Series $\mathrm{F}$ is the so-called Fibonacci series; its first two terms are o and $I$, then each succeeding term is the sum of the two preceding ones. This series is given by the proportions of heterozygotes $A a$ in various systems of inbreeding (q.v.).

Series $G$ is formed by beginning at o, doubling, then adding or subtracting $I$,- -alternately for the successive terms,--beginning with addition. Thus, twice $O$, plus $I$, gives $I$; twice I minus $I$ gives $I$; twice I plus I gives 3, etc. 'The same series is obtained if we begin with I, double, and first subtract $I$, next time adding I, etc.; but the first term of the series so obtained will be the second term of G. It may be observed that the difference between any term of $G$ and the corresponding term of $B$ always forms the next term of $G$. Thus if we subtract each term of $G$ from the corresponding term of $B$, we obtain anew the series $G$, though with each term moved one place to the right. Further, the sums of the corresponding terms of $G$ and $B$ give again the series $G$, though now with each term moved two places to the right. This peculiar series $G$ is characteristic for the results with sex-linked factors.

The remaining series of the table are combinations of those already mentioned (as shown in the second column of the table, headed "How formed"). Thus, series $\mathrm{H}$ is given by subtracting each term of $\mathrm{F}$ from the corresponding one of $G$; series $I$ by subtracting from $B$ the corresponding terms of both $F$ and $G$. Series $J$ is obtained by subtracting from a given term of $B$ the next succeeding term of $F$, (that is, $\mathrm{J}=\mathrm{B}_{n}-\mathrm{F}_{n+1}$ ). In the same way, $\mathrm{K}$ is formed by subtracting from any term of $B$ the second succeeding term of $F$. Series $L$ is another combination of $\mathrm{B}, \mathrm{F}$ and $\mathrm{G}$. Series $\mathrm{K}$ and $\mathrm{M}$ are obtained when parents of the type $A A \times A a$ are inbred. 
The table gives the first 20 terms of each series. At the left is, in each case, a letter which will be employed as a designation of the corresponding series. Before each series is given likewise a formula or statement showing, in the first seven series, how any term of the series is derived from the preceding one,- - the preceding term being called $\mathrm{x}$. Thus, in series $C, 2 x+I$ signifies that any term is formed by doubling the previous term and adding $I$. In the remaining six series this formula shows how any given term of the specified series is obtained from the other series. Thus, each term of $\mathrm{H}$ is obtained by subtracting the corresponding term of series $\mathrm{F}$ from the same term of series $\mathrm{G}$. After the last term of many of the series is given another formula or explanation showing as a rule how any given term of this series may be otherwise designated; thus the fifth term of series $\mathrm{C}$ is equal to $2^{5}-\mathrm{I}$ (or $3 \mathrm{I}$ ). In some cases certain other information is here given.

Any term of any series is designated by the aid of the letter representing the series, with the corresponding term of the series $n$ as a subscript. Thus, $\mathrm{C}_{1}$ signifies the first term of $\mathrm{C}(=\mathrm{I}) ; \mathrm{F}_{6}$ is $8 ; \mathrm{J}_{9}$ is 475 ; $B_{0}$ is $I$, etc. In general, if $n$ represents a given number, $D_{n}$ is the corresponding term of series $D$, etc. Such expressions as $F_{n+2}$ signify such a term of series $\mathrm{F}$ as is found by adding 2 to the given number $n$; thus if $n$ is $5, \mathrm{~F}_{n+2}$ is $\mathrm{F}_{7}$ (= $\mathrm{I} 3$ ).

In giving results of breeding where these series are involved, there will be given first a general expression showing what terms of the series correspond to the results of $n$ successive breedings of the given type; this will be followed by the first three or four fractions so derived (corresponding to the values $\mathrm{I}, 2,3,4$, etc., of $n$ ). For example, under paragraph (45), we find:

$$
\text { , } A A=\frac{\mathrm{J}_{n}}{\mathrm{~B}_{n+1}} ; \frac{1}{4}, \frac{2}{8}, \mathrm{1}^{\frac{5}{6}} \ldots \ldots . \frac{1}{2} ; A A_{1 \mathrm{i}}=.490 .
$$

This signifies that after one inbreeding the proportion of the population having the constitution $A A$ is $1 / 4$; after two it is $\frac{2}{8}$, after three it is $\frac{5}{16}$ after four $\frac{11}{3}$, and in general after $n$ inbreedings it is the $n$th term of the series $\mathrm{J}$, divided by the $(n+\mathrm{I})$ th term of the series $\mathrm{B}$, so that after ro inbreedings it is $\frac{J_{10}}{B_{11}}$ or $\frac{985}{2048}$. (See page 63 for further explanation.)

THE RATE OF CHANGE IN THE DIVERSE SYSTEMS OF BREEDING

Working out the concrete results for a single pair of characters, it is found that in most or all the systems of breeding there is a tendency to 
approach a constant set of proportions after a large number of generations. In most cases these constant proportions or limits are (theoretically) never actually reached, although the difference between them and the actual proportions may be made less than any designated fraction. Thus, in random mating of sex-linked characters, when the original parents are $A A$ and $a-\left(\right.$ see $\left.\left(5^{8}\right)\right)$, the proportion of males that are $A-$ approaches $\frac{2}{3}$, of those that are $a$-approaches $1 / 3$; oi the females, $A A$ and $A a$ each approach $\frac{4}{9}$, while $a a$ approaches $\frac{1}{9}$; but these limiting proportions are never actually reached. In brother by sister mating (29), the proportions of $A A$ and $a a$ each approach $1 / 2$, while that of $A a$ approaches 0 .

For any particular case, after the formulae have been worked out, as given in the text, the proportions which any particular class approach may be obtained from a table giving once for all the limits approached by the various series of table $\mathrm{I}$, when divided by the corresponding terms of the series B, - since most of our results take the form of such fractions. For example, in the mating of brother by sister, sex-linked character (69), in the males the proportion of $A-$ is $\frac{\mathrm{G}_{n+1}}{\mathrm{~B}_{n}}$, and this fraction approaches the value $\frac{2}{3}$; of $a$ the proportion is $\frac{\mathrm{G}_{n}}{\mathrm{~B}_{n}}$, which approaches the value $\mathrm{I} / 3$; in the females $A A$ is $\frac{\mathrm{I}_{n+1}}{\mathrm{~B}_{n+1}}$, which approaches the limit $\frac{2}{3}$, aa is $\frac{\mathrm{H}_{n+1}}{\mathrm{~B}_{n+1}}$, approaching the limit $1 / 3 ; A a$ is $\frac{\mathrm{F}_{n+1}}{\mathrm{~B}_{n}}$, whose limit is 0 .

We therefore give in table 2 the limits approached by the fractions obtained when the diverse series are divided by $B$.

Table 2 includes also certain other data of importance. One needs to know, not only the theoretical limit approached, but also how long a series of breedings is required before the limit is practically attained. In most cases there is at first rapid progress toward the limiting, value, and it is attained within say I percent after only 6 or 7 generations; thereafter progress is so extremely slow as to be practically negligible. This is the condition already pointed out by PEARL (1913) for certain systems of inbreeding. For example, in self-fertilization, where the original cross was $A A \times A a$ (see (26)), the proportion of $A A$ gives the series $\frac{\mathrm{E}_{n+2}}{\overline{\mathrm{B}}_{n+2}}$ the limit of which is $3 / 4$, or 75 percent. But after five selffertilizations $A A$ has already reached the proportion 74.2 percent, so that further breeding in this way has little further effect. In table 2 


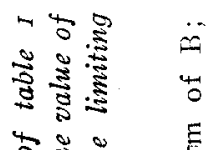

ชั

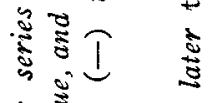

ט

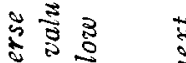

嗾

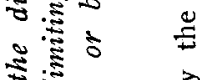

至定

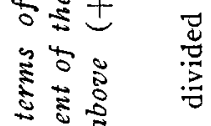

ह

$\approx$ ह

$\circ \cdot \overline{3}$

. क्षे

.

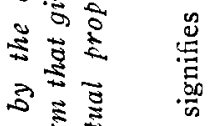

ริ

.

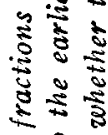

का

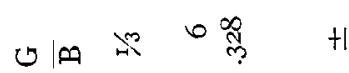

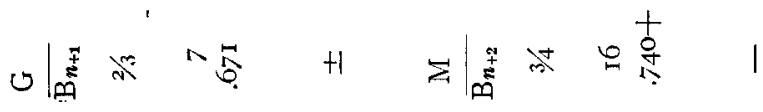

山|

工苞

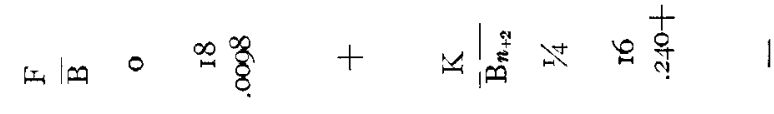

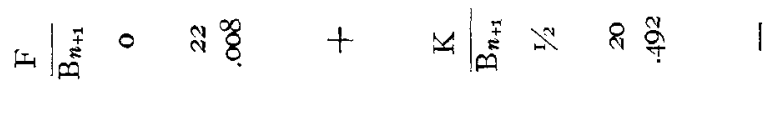

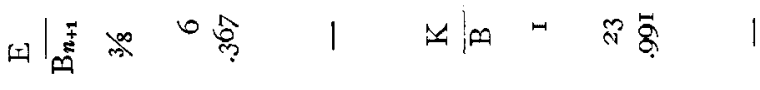

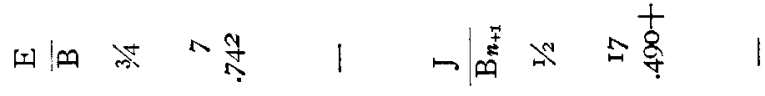

ค|

is $\frac{\pi}{8} \frac{\pi}{8}$

$\because \therefore \frac{5}{3}$

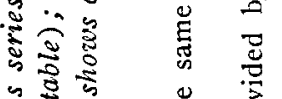

के के छ

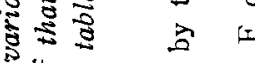

ङड

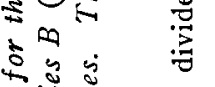

ह

U

के

计蛙

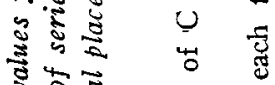

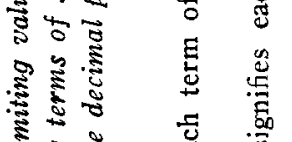

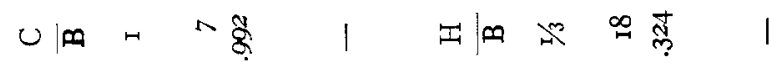

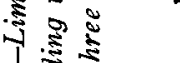

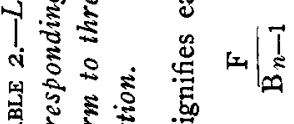

言泀吉士自

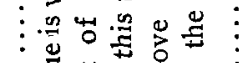

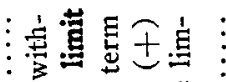

泀若出空I。

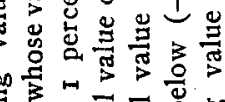

कू है Uा

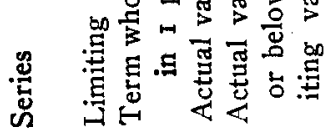

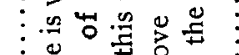

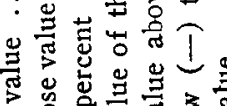

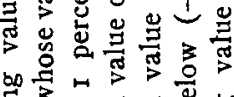




we give, therefore, the earliest term of the series in which the difference between the final or limiting proportion and the proportion actually attained is less than I percent of the total. From this we can readily determine the number of generations required in any system of breeding to bring the actually attained proportion within I percent of the theoretical limit.

In most of the series of table 2, the value attained at any term is below the theoretical limit. In the series formed by the division of $\mathrm{D}$ and of $F$ by $B$, the actual value is always above the limit,--the limit being approached from above. In the series formed by dividing $G$ by $B$, the actual values are alternately below and above the limiting value, the difference decreasing in later terms. These relations are shown in table 2 by use of the signs + and - , as also of \pm .

The information summarized in table 2 is given more precisely and fully in the text, under the proper paragraph. After giving the general formula and the first few terms of the series to which it gives rise, the limiting value of the proportions is given, and this is followed by the actual value of the proportions for the smallest number of generations in which the proportion attained is within I percent of the limiting value (see explanation on page 63 ).

\section{DERIVATION OF THE FORMULAE}

In the actual working out, the path to most of the formulae has been long and indirect; frequently with many windings and digressions. To give in detail the method of working out each formula would swell the paper beyond all bounds. I am compelled, therefore, in most cases, to content myself with giving the actual formulae, leaving their correctness to the test of time. In a few cases some indications are given of special points requiring attention in the working out. The general method of work is something as follows:

One begins with the formulae of the assumed parents (as $A A \times A a$ ), forms the gametes, and mates them (all symbolically of course), obtaining the results for generation I. One may continue this for five or six generations, getting the proportions of each sort of individuals for each generation. The work is shortened by obtaining at the beginning a formula for transforming the proportions for one generation into those for the next (such a formula as is given in (8)). After obtaining the results for five or six generations, one examines the series of results, and the method of derivation, to discover general relations that may 
give a regular series, or be embodied in a formula. Curiously, the study of the actual procedure in deriving results is usually of little assistance in determining the nature of the series or of the general formula. One must, as a rule, take the series of results as finished products, and make an independent study of them, endeavoring by processes of trial to fit them to some series or to some formula. It is here that there is scope for ingenuity; a given series of results may resist for weeks the discovery of the law that unites them. After the system of fundamental series, given in table I, had been discovered, it was usually found possible to fit the results to some combination of these; but of course the work was three-fourths done before this system was worked out. The series of results as one obtains them are often in perplexing form. For example, in (43), the proportions of $A A$ are for five generations successively $1 / 2,3 / 8 \frac{1}{2}, 1 / 2, \frac{3}{6} \frac{5}{4}$. It is difficult to discover the general law underlying such a series, though it turns out that there is one.

After a law or regular series is obtained that fits the first five or six generations, the law is applied to give the results for three or four generations more. These results are then tested by the actual detailed working out (symbolic formation of gametes and their mating, etc.) for these same later generations; if the formula has given the correct results, it is assumed to be a general formula.

More rarely, the formula is obtained by generalizing the actual procedure in the symbolic formation of gametes and their mating according to the given method.

METHOD OF PRESENTATION ; DIRECTIONS FOR USE OF THE FORMULAE

The ideal method of presentation of the formulae would be in tables, but this is impracticable, owing to the somewhat complex conditions that must be specified. They will, therefore, be presented in a series of numbered paragraphs. Any single pair of alternative factors will be represented by $A$ and $a$, in the three possible combinations $A A$, $a a$ and $A a$. The number of successive times. any given sort of breeding has occurred (that is, the number of generations that the system has been followed) will be called $n$. The formulae are designed to show the relative proportions of the population formed by each of the three sorts of individuals ( $A A, a a$ and $A a$ ) after any number $n$ of breedings of the designated sort (from these the relative proportions of homozygotes and heterozygotes; of dominants and recessives, are of course, at once 
obtainable); also the limit which each proportion tends to approach as breeding is continued; and the smallest number of generations that breeding must be continued before the actual proportions come within one percent of the limiting value. The information given will best be understood from an example. In (3I), page 72 , we find:

$$
A A=\frac{\mathrm{M}_{n+1}}{\mathrm{~B}_{n+3}} ; \frac{9}{16}, \frac{19}{32}, \frac{40}{64} \ldots . . \frac{3}{4} . \quad A A_{15}=.740 \mathrm{r} .
$$

Here we have:

1. The general formula for the proportion of the population having the constitution $A A$, after any number $n$ of breedings of the sort set forth. This general formula, $\frac{\mathrm{M}_{n+1}}{\mathrm{~B}_{n+3}}$, enables one to find from table I, page 56, the proportion of $A A$ after any particular number of generatjons $(n)$; thus, after I2 such matings ( $n=\mathrm{I} 2$ ), the proportion of $A A$ is $\frac{\mathrm{M}_{13}}{\mathrm{~B}_{15}}=\frac{23}{3} \frac{3966}{2} \frac{66}{76}=.73 \mathrm{I}$.

2. Immediately following the general formula are the first three terms of the series given by the formula; that is, the proportions when $n$ is $\mathrm{I}, 2$, and 3 ; these are in this case $\frac{9}{16}, \frac{19}{32}, \frac{40}{64}$.

3. Following these, after a series of dots, is the limiting value which these proportions approach. Thus, in this case, after a very large number of inbreedings the proportion of the population that are $A A$ would be very near $3 / 4$. This limiting value is never actually attained, but the difference between it and the actual value may be made smaller than any designated value.

4. Finally is given, in the form $A A_{15}=.740 \mathrm{O}$, the lowest number, $\mathrm{I}_{5}$, of inbreedings necessary to bring the actual proportion within $\mathrm{I}$ percent of the limiting value, together with the actual proportion (.740I) in this generation. Thus, in this case, the formula shows that we must continue inbreeding 15 generations before the individuals $A A$ form within I percent of the possible $3 / 4$ of the population, that at that time they form .740I of the population, and that if we continue to inbreed we make very little further progress, and can never in this way cause $A A$ to form quite .75 of the population.

Similarly, in paragraph (3I), page 72 , we find given the general formula and the first three terms of the series for the proportions of the population formed by $a a$ and $A a$. We find that $a a$ approaches gradually the limit $1 / 4$, while $A a$ comes nearer and nearer to o, although these limits are never quite reached. We find further that after ${ }_{15}$ inbreedings $a a$ comes within I percent of forming $1 / 4$ of the population, its actual 
proportion at that time being .240I. After I9 generations $A a$ comes within I percent of being 0 , its actual proportion being then .008 .

In some cases the general formula given is the same as that in a preceding paragraph. In such a case the first terms of the series and the limiting values are not repeated; they should be sought in one of the immediately preceding paragraphs. This is the case, for example, in $(36)$; the terms and limit are given in (35).

For some cases the general formula involves other variables besides $n$, so that a concrete series of terms can not be given and no general limit can be assigned. Such are found in (2), (8), etc. These and various other methods of presentation are self-explanatory.

\section{TyPICAL FACTORS, NOT SEX-LINKED}

\section{a. Random mating}

(1) The population at the beginning consists of individuals $A A$ and $a a$, in equal numbers. These mate at random ( $A A$ with $A A$ or with $a a$, etc.).

In any later generation the population consists of :

$$
\begin{aligned}
& \mathrm{I} / 4 A A+\mathrm{I} / 4 a a+\mathrm{I} / 2 A a \\
& 1 / 2 \text { Homozygotes }+1 / 2 \text { heterozygotes } \\
& 3 / 4 \text { Dominants }+1 / 4 \text { recessives. }
\end{aligned}
$$

(2) If the proportions of the two kinds are at the beginning not equal, but there are $r A A$ to $t a a$, in later generations the population consists of :

$$
r^{2} A A+t^{2} a a+2 r t A a
$$

The condition given in (I) is only a special case of this, where $r$ and $t$ are both I.

(3) If the population at the beginning are all $A a$, in later generations the constitution is the same as that set forth under ( $I$ ).

(4) The population at the beginning consists of individuals $A A$ and $A a$, in equal numbers, mating at random ( $A A$ with $A A$ or $A a$, etc.) In later generations the population consists of :

$$
\begin{aligned}
& \frac{9}{16} A A+\frac{1}{16} a a+\frac{6}{16} A a \\
& 5 / 8 \text { Homozygotes }+3 / 8 \text { heterozygotes } \\
& \frac{15}{16} \text { Dominants }+\frac{1}{16} \text { recessives }
\end{aligned}
$$


(5) The proportions of $A A$ and $A a$ at the beginning are not equal, there being $r A A$ to $s A a$. In later generations the population is

$$
\frac{(s+2 r)^{2} A A+s^{2} a a+2 s(s+2 r) A a}{4(s+r)^{2}} .
$$

Case (4) is only a special case of this.

(6) If the population at the beginning is $a a$ and $A a$, the results are the same as given under (4) and (5), save that the proportions for $a a$ and $A A$ are interchanged, that for $A a$ remaining the same.

(7) If a breeding experiment begins with a single pair ( $A A$ and $a a$, or $A A$ and $A a$, for example), and these are mated, this first mating is not a random mating, but a defined one, and the later conditions specified in (I) and (4) are not reached till after an actual random mating; that is, until the generation $\mathrm{f} 2$; they persist thereafter, so long as the matings are random.

(8) The conditions thus far specified are all special cases of the general condition that at the beginning of random mating the population consists of $r A A+t a a+s A a$. If $r, t$ and $s$ are diverse and none of them are zero, the constitution of the population is not uniform in later generations, but changes with the number of random matings that occur. If the constitution of any generation $n$ is $r A A+t a a+s A a$ then the constitution of the next following generation is:

$$
\begin{aligned}
& A A=(s+2 r)^{2} . \\
& a a=(s+2 t)^{2} . \\
& A a=2(s+2 r)(s+2 t) .
\end{aligned}
$$

Thus, if at the beginning of random mating the proportions are $3 A A$ $+4 a a+5 A a$, in the next generation the proportions will be:

$$
\begin{aligned}
A A & =(5+2 \times 3)^{2}=\mathrm{II}^{2}=\mathrm{I} 2 \mathrm{I} \\
a a & =(5+2 \times 4)^{2}=\mathrm{I}^{2}=\mathrm{I} 69 \\
A a=2 \times \mathrm{II} \times \mathrm{I} 3= & 286 .
\end{aligned}
$$

The proportions for succeeding generations can be found in the same way. I have not been able to obtain a serviceable formula giving directly the proportions for any later generation, without working out the proportions for the intervening generations.

(9) If $r$ and $t$ are equal (whatever the value of $s$ ), the later proportions are those given under ( $\mathrm{I}$ ).

(Io) If $s$ is zero, the condition set forth under (2) is realized.

(II) If $r$ or $t$ is zero, the condition set forth under (5) or (6) is realized. 
(I2) If $r$ and $t$ are both zero, the condition given in (3) is realized. The results for (9), (IO), (II) and (I2), as given in (I), (2). (5), (6) and (3), flow directly from the relations set forth under (8) when the proper substitutions are made. These relations (8) are the fundamental ones for discovering all the rest.

b. Assortative mating,-dominants with dominants, recessives with recessives

If in pure stocks dominants mate with dominants, recessives with recessives, of course the stocks simply remain pure. The problem of how such breeding will result has point only after crossing has occurred, so that heterozygous dominants are present. Therefore, it is necessary to specify in each case what cross or what other sort of mating has occurred before the assortative mating begins.

In such assortative mating after a cross, $A A$ mates with $A A$ or with $A a$, while aa mates only with $a a$.

( I3) The population at the beginning consists of $A A$ and $a a$ in equal numbers; these mate at random, producing $1 / 4 A A+1 / 4 a a+1 / 2 A a$; assortative mating then occurs among these progeny.

There are certain pitfalls to be avoided in working out the proportions in later generations. In the generation before assortative mating occurs, the proportion of dominants $(A A+A a)$ is $3 / 4$, while the recessives are $\mathrm{r} / 4$. The essential point to keep in mind is that in the next generation the progeny produced by the dominants will be $3 / 4$ of all the progeny produced, while those from the recessives (which will all be recessive) will be $\mathrm{s} / 4$. We have then merely to find out what will be the relative proportions of $A A, a a$ and $A a$ among the progeny of the dominants; to get their proportions of the total we must multiply these proportions from dominants by $3 / 4$. For aa the proportion so obtained (derived from the dominants) must be added to $1 / 4$, to give the proportion of the total progeny that are recessive. Proceeding in this way, we find that after any number $n$ of successive assortative matings, the proportions of the three classes are:

$$
\begin{aligned}
& A A=\frac{n+\mathrm{I}}{2 n+4} ; \frac{2}{6}, \frac{3}{8}, \frac{4}{10} \ldots \ldots \frac{1}{2} . A A_{48}=.490 . \\
& a a=\frac{n+1}{2 n+4} . \\
& A a=\frac{1}{n+2} ; \frac{1}{3}, \frac{1}{4}, \frac{1}{5} \ldots \ldots \circ . A a_{98}=.010 .
\end{aligned}
$$




$$
\begin{aligned}
& \text { Homozygotes }=\frac{n+\mathrm{I}}{n+2} ; \text { heterozygotes }=\frac{\mathrm{I}}{n+2} . \\
& \text { Dominants }=\frac{n+3}{2 n+4} ; \text { recessives }=\frac{n+\mathrm{I}}{2 n+4} .
\end{aligned}
$$

(14) In the more general case the poptlation at the beginning is $r A A$ to $t a a$; which mate at random, assortative mating occurring in their progeny.

By (2) the progeny derived from the original parents is

$$
r^{2} A A+2 r t A a+t^{2} a a \text {. }
$$

Now dominant mates with dominant, recessive with recessive. After $n$ successive assortative matings, the population is:

$$
\begin{aligned}
A A & =\frac{r(r+n t)}{(r+t)(r+n t+t)} . \\
a a & =\frac{(n+1) t^{2}}{(r+t)(r+n t+t)} . \\
A a & =\frac{2 r t}{(r+t)(r+n t+t)} .
\end{aligned}
$$

(I5) In the still more general case the population before a given assortative mating, is composed of $r A A+\mathrm{t} a a+s A a$. In the next generation (after the assortative mating), the proportions are:

$$
\begin{aligned}
A A & =\frac{(2 r+s)^{2}}{4(r+s)(r+s+t)} . \\
a a & =\frac{s^{2}+4 r t+4 s t}{4(r+s)(r+s+t)} . \\
A a & =\frac{4 r s+2 s^{2}}{4(r+s)(r+s+t)} .
\end{aligned}
$$

By continued use of these formulae (substituting at any generation the proportions of $A A$ for $r$, of $a a$ for $t$, of $A a$ for $s$ ), the results for any number of generations of assortative matings may be obtained: These relations are the fundamental ones for working out the formulae given in the following paragraphs (as well as in (13)).

(I6) The population at the beginning is all $A a$; assortative mating occurs. After $n$ such matings:

$$
\begin{aligned}
A A & =\frac{n}{2 n+2} ; \frac{1}{4}, \frac{2}{6}, \frac{3}{8} \ldots \ldots \frac{1}{2} . A A_{48}=.490 . \\
a a & =A A . \\
A a & =\frac{1}{n+1} ; \frac{1}{2}, \frac{1}{3}, \frac{1}{4} \ldots . .0 . \quad A a_{99}=.010 .
\end{aligned}
$$

Genetics 1: Ja 1916 
(If the experiment begins by a cross of $A A$ with $a a$, of course the first assortative mating occurs thus when all are $A a$ ).

(I7) The population at the beginning consists of $A A$ and $A a$ in equal numbers. After $n$ assortative matings (including among these the first mating, where all are dominants), the proportions are as follows:

$$
\begin{aligned}
& A A=\frac{3 n+6}{4 n+12} ; \text { thus } \frac{9}{15}, \frac{12}{20}, \frac{15}{24}, \frac{18}{28} \ldots \ldots+\frac{3}{4} . A A_{72}=.740 . \\
& a a=\frac{n}{4^{n+12}} ; \text { thus } \frac{1}{16}, \frac{2}{20}, \frac{3}{24}, \frac{4}{28} \ldots \ldots \frac{1}{4} . \quad a a_{72}=.240 . \\
& A a=\frac{6}{4 n+12} ; \text { thus } \frac{6}{16}, \frac{6}{20}, \frac{6}{24}, \frac{6}{28} \ldots \ldots \text { o. } A a_{147}=.010 . \\
& \text { Dominants }=\frac{3 n+12}{4 n+12} ; \quad \text { recessives }=\frac{n}{4 n+12} .
\end{aligned}
$$

(18) At the beginning we have $a a$ and $A a$ in equal numbers. If these cross, giving again $a a+A a$, there is no opportunity for assortative mating. But if instead of crossing, they mate at random (producing I $A A+9 a a+6 A a)$, then assortative mating begins; - after $n$ such matings :

$$
\begin{aligned}
& A A=\frac{3^{n+1}}{12 n+16} ; \text { thus } \frac{4}{28}, \frac{7}{40}, \frac{10}{52} \ldots \ldots+\frac{1}{4} . A A_{24}=.240 \mathrm{r} . \\
& a a= \frac{9(n+\mathrm{I})}{12 n+16} ; \text { thus } \frac{18}{2} \frac{8}{8}, \frac{27}{4}, \frac{36}{52} \ldots \ldots \frac{3}{4} . \quad a a_{24}=.740 \mathrm{I} . \\
& A a= \frac{6}{12 n+16} ; \text { thus } \frac{6}{28}, \frac{6}{40}, \frac{6}{52} \ldots \ldots . . \quad A a_{49}=.0099 . \\
& \quad \text {. Selection of the dominants alone }
\end{aligned}
$$

Only dominants are bred, the recessives being rejected in each generation. In this case, as in assortative mating, some sort of a cross must have occurred at the beginning, otherwise no problem is involved.

(19) The population is at first $A A$ and $a a$ in equal numbers; these mate at random, producing $1 / 4 A A+\mathrm{I} / 4 a a+\mathrm{r} / 2 A a$; the dominants alone are bred (at random among themselves). After $n$ such breedings, the population is:

$$
\begin{aligned}
A A & =\frac{(n+1)^{2}}{(n+2)^{2}} ; \frac{4}{9}, \frac{9}{16}, \frac{16}{25} \ldots \ldots \text { I. } A A_{198}=.990+. \\
a a & =\frac{1}{(n+2)^{2}} ; \frac{1}{9}, \frac{1}{16}, \frac{1}{25} \ldots \ldots \circ . \quad a a_{8}=.010 . \\
A a & =\frac{2(n+1)}{(n+2)^{2}} ; \frac{4}{9}, \frac{6}{16}, \frac{8}{25} \ldots \ldots \text {. } . \quad A a_{199}=.0099 .
\end{aligned}
$$

(20) In the more general case, the population at first consists of 
$r A A$ to $t a a$, which mate at random (giving according to (2) $r^{2} A A+$ $\left.t^{2} a a+2 r t A a\right)$; then dominants alone are bred. After $n$ such successive selections, the population is:

$A A=\frac{(r+n t)^{2}}{\left(r+(n+I t)^{2}\right.} . \quad a a=-\frac{t^{2}}{(r+(n+I) t)^{2}} . \quad A a=\frac{2 t(r+n t)}{(r+(n+I) t)^{2}}$.

(2I) In the still more general case, before a given selective breeding the population is $r A A+t a a+s A a$.

Of course the $t$ aa do not breed at all, and play, therefore, no rôle. After the next selection of dominants:

$$
A A=\frac{(2 r+s)^{2}}{4(r+s)^{2}} . \quad a a=\frac{s^{2}}{4(r+s)^{2}} . \quad A a=\frac{2 s(2 r+s)}{4(r+s)^{2}} .
$$

By continuation, the results for any number of generations can be worked out.

(22) If at first $A A$ is crossed with aa, giving $A a$, and then dominants alone are bred, the result is the same as in (19), save that any given proportion is reached one generation later. That is,

$$
A A=\frac{n^{2}}{(n+\mathrm{I})^{2}} . \quad a a=\frac{\mathrm{I}}{(n+\mathrm{I})^{2}} . \quad A a=\frac{2 n}{(n+\mathrm{I})^{2}} .
$$

(23) At the beginning $a a$ is crossed with $A a$, giving $A a+a a$; only dominants are bred. This gives the same results as (22).

(24) $A A$ is crossed with $A a$, giving $A A+A a$; then only dominants are bred (at random among themselves). This gives the same series of results as do (19) and (22), but any given result comes one generation sooner than in (19), and two generations sooner than in (22). That is, after $n$ breedings of dominants alone:

$$
A A=\frac{(n+2)^{2}}{(n+3)^{2}} . \quad a a=\frac{\mathrm{I}}{(n+3)^{2}} . \quad A a=\frac{2 n+4}{(n+3)^{2}} .
$$

\section{d. Self-fertilization}

Some sort of a cross must have occurred before self-fertilization begins, otherwise, of course, the stock remains constant.

(25) Original cross, $A A$ by $a a$, giving all $A a$. Thereafter all breeding is by self-fertilization. In this case $A A$ and $a a$ each give the series $\mathrm{C}$ (table I), while $A a$ is 2 in every generation. That is, after $n$ selffertilizations :

$$
\begin{aligned}
& A A=\frac{\mathrm{C}_{n}}{\mathrm{~B}_{n+1}}\left(=\frac{2^{n}-\mathrm{I}}{2^{n+1}}\right) ; \frac{1}{4}, \frac{3}{8}, \frac{7}{1^{7}} \ldots \ldots . \frac{1}{2} \cdot A A_{6}=.492 . \\
& a a=-\frac{\mathrm{C}_{n}}{\mathrm{~B}_{n+1}}\left(=\frac{2^{n}-\mathrm{I}}{2^{n+1}}\right) . \\
& A a=\frac{2}{2^{n+1}}\left(=\frac{\mathrm{I}}{2^{n}}\right) ; \frac{1}{2}, \frac{1}{4}, \frac{1}{8} \ldots \ldots \text {. } A a_{7}=.008 .
\end{aligned}
$$


(26) Original cross $A A$ by $A a$; thereafter self-fertilization. In this case $A A$ gives the series $\mathrm{E}$, beginning at 5 ; $a a$ gives the series $\mathrm{C}$, beginning at I, while $A a$ is always 2. After $n$ self-fertilizations:

$$
\begin{aligned}
& A A=\frac{\mathrm{E}_{n+2}}{\mathrm{~B}_{n+2}}\left(=\frac{2^{n+1}+2^{n}-\mathrm{I}}{2^{n+2}}\right) ; \frac{5}{8}, \frac{11}{16}, \frac{23}{32}, \frac{47}{64} \ldots \ldots . .3 A_{5}=.742 . \\
& \alpha a=\frac{\mathrm{C}_{n}}{\mathrm{~B}_{n+2}}\left(=\frac{2^{n-1}}{2^{n+2}}\right) ; \frac{1}{8}, \frac{3}{16}, \frac{7}{32}, \frac{15}{64} \ldots \ldots \frac{1}{4} \cdot a a_{5}=.242 . \\
& A a=\frac{2}{\mathrm{~B}_{n+2}}\left(=\frac{\mathrm{I}}{\mathrm{B}^{n+1}}\right)\left(=\frac{\mathrm{I}}{2^{n+1}}\right) ; \frac{1}{4}, \frac{1}{8}, \frac{1}{14}, \frac{1}{64} \ldots \ldots . \quad A a_{6}=.008 .
\end{aligned}
$$

(27) The population at the beginning shows the typical Mendelian proportions, $1 / 4 A A+1 / 4 a a+1 / 2 A a$. Now self-fertilization begins and is continued. In this case we obtain the same three series as in (25), but beginning one term farther; that is, after $n$ self-fertilizations:

$$
\begin{aligned}
A A & =\frac{\mathrm{C}_{n+1}}{\mathrm{~B}_{n+2}}\left(=\frac{2^{n+1}-\mathrm{I}}{2^{n+2}}\right) ; \frac{3}{8}, \frac{7}{16}, \frac{15}{3} \ldots \ldots \frac{1}{2} . A A_{5}=.492 . \\
a a & =\text { same as } A A . \\
A a & =\frac{2}{\mathrm{~B}_{n+2}}\left(=\frac{2}{2^{n+2}}=\frac{\mathrm{I}}{2^{n+1}}\right) ; \frac{1}{4}, \frac{1}{8}, \frac{1}{116} \ldots . . \quad A a_{6}=.008 .
\end{aligned}
$$

(28) The three cases so far considered are particular instances of the general case that the population consists at the beginning of $r A A+t$ $a a+s A a$. Then after $n$ self-fertilizations:

$$
\begin{aligned}
& A A=\frac{r\left(2^{n+1}\right)+s\left(2^{n}-1\right)}{(r+s+t) 2^{n+1}} . \quad a a=\frac{t\left(2^{n+1}\right)+s\left(2^{n}-1\right)}{(r+s+t) 2^{n+1}} . \\
& A a=\frac{2 s}{(r+s+t) 2^{n+1}} .
\end{aligned}
$$

For any particular constitution of the population at the beginning the results after any number of self-fertilizations will readily be obtained by making the proper substitutions for $r, s, t$ and $n$ in these formulae. For example, if the original population is $2 A A+4 a a+3 A a$, after three self-fertilizations:

$$
A A=\frac{53}{144} . \quad \alpha a=\frac{91}{144} . \quad A a=\frac{6}{144} .
$$

Inbreeding may occur according to various diverse systems, and in each case the outcome depends of course on the constitution of the parents originally crossed; or, in the case of an entire population, on the constitution of the population at the time inbreeding begins. In our formulae the number of inbreedings $(n)$ includes only the actual matings of related individuals, not the cross that precedes such matings. The 
Fibonacci series (F, table $I$ ) is the key series in most cases of inbreeding.

\section{e. Brother and sister mating}

When the original cross is either $A A \times a a$, or $A A \times A a$, or $a a \times A a$, or $A a \times A a$, followed by brother $\times$ sister mating among the progeny, the proportions of heterozygotes $A a$ form in successive generations a series of fractions of which the terms of the Fibonacci series ( $F$ in table $I$ ) are the numerators, while the denominators are certain powers of 2 (forming thus our series $B$ ). In the different original matings the series for $A a$ begins at different points in the $\mathrm{F}$ series. Where the original cross is $A A \times a a$ or $A a \times A a$, the proportions of $A A$ (or of aa) are $1 / 2$ of the difference between $I$ and the fraction showing the proportion of the heterozygotes $A a$. Thus, if the heterozygotes are $3 / 8$, the proportion for $A A$ is $I / 2(\mathrm{I}-3 / 8)=\frac{5}{16}$; the proportion of $a a$ is the same.

Where the original cross is $A A$ by $A a$, the proportion for the sum of $(A A+a a)$ is $(\mathrm{I}-A a)$, but $(A A+1 / 2 A a)$ is always equal to three times $(a a+\mathrm{r} / 2 A a)$, so that $A A=3 a a+A a$. If the original cross is $a a \times A a$, the proportions are reversed; so that $a a=3 A A+A a$. It results from these relations that the proportions for $A A$ and $a a$ can readily be found when those for $A a$ are known. When the crosses are $A A \times a a$, or $A a$ by $A a$, the proportions for $A A$ and $a a$ give our series $\mathrm{J}$. When the first cross is $A A$ by $A a$, the proportions of $A A$ give the series $\mathrm{M}$, while $a a$ gives the series $\mathrm{K}$. If the first cross is $a a$ by $A a$, then $a a$ gives $\mathrm{M}$, while $A A$ gives $\mathrm{K}$. We shall now for reference give the formulae for each of the cases.

(29) Original cross $A A$ by $a a$. After $n$ brother by sister matings:

$$
\begin{aligned}
A A & =\frac{\mathrm{J}_{n}}{\mathrm{~B}_{n+1}} ; \frac{1}{4}, \frac{2}{8}, \frac{5}{16}, \frac{11}{32} \ldots \ldots \frac{1}{2} . A A_{17}=.490+. \\
a \alpha & =\frac{J_{n}}{\mathrm{~B}_{n+1}} . \\
A a & =\frac{\mathrm{F}_{n+1}}{\mathrm{~B}_{n}} ; \frac{1}{2}, \frac{2}{4}, \frac{3}{8}, \frac{5}{16} \ldots . . . A \alpha_{21}=.008+.
\end{aligned}
$$

(30) Original cross $A a \times A a$. After $n$ inbreedings:

$$
\begin{aligned}
A A & =\frac{\mathrm{J}_{n+1}}{\mathrm{~B}_{n+2}} ; \frac{2}{8}, \frac{5}{16}, \frac{11}{32}, \frac{24}{64} \ldots \ldots \frac{1}{2} . A A_{16}=.490+. \\
a a & =A A . \\
A a & =\frac{\mathrm{F}_{n+2}}{\mathrm{~B}_{n+1}} ; \frac{2}{4}, \frac{3}{8}, \frac{5}{16}, \frac{8}{32} \ldots \ldots \circ . A a_{20}=.008 .
\end{aligned}
$$


(3I) Original cross $A A \times A a$. After $n$ inbreedings:

$$
\begin{aligned}
& A A=\frac{\mathrm{M}_{n+1}}{\mathrm{~B}_{n+3}} ; \frac{9}{16}, \frac{19}{32}, \frac{40}{64} \ldots \ldots .3 .4 A_{15}=.740 \mathrm{I} . \\
& a a=\frac{\mathrm{K}_{n+1}}{\mathrm{~B}_{n+3}} ;{ }_{16}^{1}, \frac{3}{32},{ }_{64}^{8} \ldots . .+240 \mathrm{I} . \\
& A a=\frac{\mathrm{F}_{n+3}}{\mathrm{~B}_{n+2}} ; \frac{3}{8}, \frac{5}{17}, \frac{8}{32} \ldots . . \quad A a_{19}=.008 .
\end{aligned}
$$

(32) Original cross $a a \times A a$. After $n$ inbreedings:

$$
\begin{aligned}
& A A=\frac{\mathrm{K}_{n+1}}{\mathrm{~B}_{n+3}} ; \frac{1}{16}, \frac{3}{32}, \frac{8}{64} \ldots \ldots \frac{1}{4} . A A_{15}=.240 \mathrm{I} \text {. } \\
& a \alpha=\mathrm{M}_{n+1} ; \underset{16}{\mathrm{~B}_{n+3}}, \frac{99}{32}, \frac{40}{64} \ldots . .3 . \quad \alpha a_{15}=.740 \mathrm{I} . \\
& A a=\frac{\mathrm{F}_{n+3}}{\mathrm{~B}_{n+2}} ; \frac{3}{8}, \frac{5}{16}, \frac{8}{32} \ldots .0 . \quad A a_{19}=.008 .
\end{aligned}
$$

(33). In a Mendelian population showing the typical proportions $\mathrm{I} / 4 A A+\mathrm{I} / 4 a a+\mathrm{I} / 2 A a$, exclusive brother $\mathrm{X}$ sister mating begins at a certain time. After $n$ such inbreedings the results are the same as given in $(30)$.

(34) It may be observed that in all cases the proportions for $A a$ form a series of fractions in which the numerator of any term is the sum of the numerators of the two preceding terms, while its denominator is double that of the preceding term. One requires therefore but to know the first two terms of any series (as given in preceding paragraphs) in order to write out the entire series as far as he desires.

Similarly, the proportions for $A A$ and $a a$ may be written out by a general rule when the first two terms in any series (as given above) are known. The rule is to double the numerator and denominator of the preceding term, and add to the numerator the sum of the two last additions so made. For example, in the series for $A A$ given under $(3 \mathrm{I})$, to obtain the second numerator 19 from 9 , one evidently doubles 9 and adds one; to get 40 from 19 , one doubles 19 and adds 2 ; therefore, to get the numerator of the next term one must double 40 and add $\mathrm{I}+$ $2=3$, giving $\frac{83}{128}$; for the next numerator one doubles 83 and adds $2+3=5$, giving $\frac{1}{2} \frac{7}{5}$, etc.

The formulae here given are simpler than the one proposed in my paper in the American Naturalist of November, r9i4.

\section{f. Mating of parent by offspring}

The inbreeding of parent by offspring is practicable in higher organisms mainly in the successive breeding back of daughters to the father. 
In this way the same individual is used repeatedly as parent in successive generations, as indicated in the diagram (figure I).

Other systems of breeding parent by offspring are conceivable, and might be carried out in lower organisms, particularly plants. Some of these will be considered for, their theoretical interest.

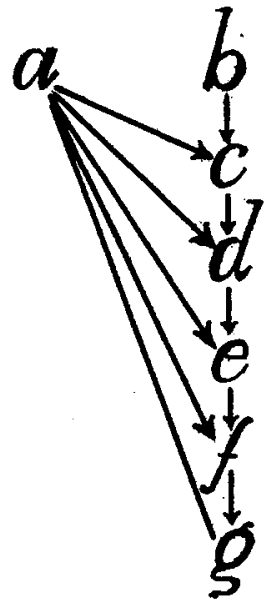

FIG. I

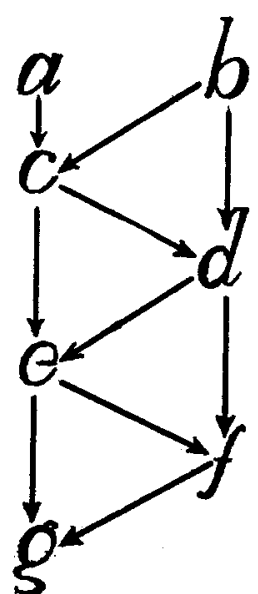

FIG. 2.

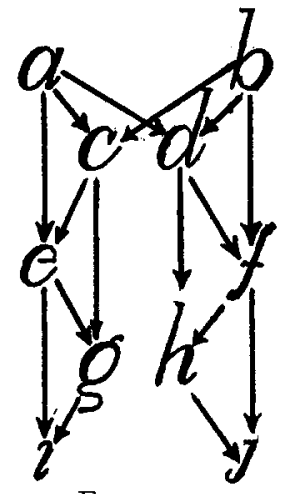

FIG. 3.

Figures I, 2 and 3.-Diagrams illustrating different conceivable systems of mating parents to offspring. The letters represent individuals; the arrows show their parentage.

FIGURE I.-Continued mating of daughters back to their father. The same father $a$ is bred from in each generation.

FIGURE 2.-The alternating system of breeding parent by offspring. Each parent saz'e one (a) is bred from twice; thrus $b$ is parent of $c$, and is also mated with $c$ to produce $d ; c$ is parent of $d$ and $e$, etc. (For simplicity's sake only one offspring is represented in each generation.)

FIGURE 3.-The alternating system of breeding parent by offspring, when both the original parents are represented to the same extent, through breeding half their progeny to one parent, half to the other. (Only one of the offspring from each parent is represented, save that two of the $\mathrm{f}_{1}$ progeny of $a$ and $b$ are shown, in order that the family may be divided into two.)

i. Same father bred to his daughters in successive generations (fig. I).

(35) Original parents $A A$ and $a a$; the progeny are always mated back to $A A$. The proportions for $A A$ give the series $\mathrm{C}$, while aa is $\mathrm{o}$, and $A a$ is $\mathrm{I}$. That is, after $n$ such inbreedings :

$$
\begin{aligned}
A A & =\frac{\mathrm{C}_{n}}{\mathrm{~B}_{n}}\left(=\frac{2^{n}-\mathrm{I}}{2^{n}}\right) ; \frac{1}{2}, \stackrel{3}{4}, \frac{7}{8} \ldots \ldots \text { I . } A A_{7}=.992 . \\
a a & =0 . \\
A a & =\frac{\mathrm{I}}{2^{n}} ; \frac{1}{2}, \frac{1}{4}, \frac{1}{8} \ldots \ldots \ldots \ldots \text { o. } A a_{7}=.008 .
\end{aligned}
$$


(36) Parents $a a \times A A$; progeny mated back to $a a$. After $n$ inbreedings :

$$
a a=\frac{\mathrm{C}_{n}}{\mathrm{~B}_{n}^{-}} . \quad A A=0 . \quad A a=\frac{\mathrm{I}}{2^{n}} .
$$

(37) Parents $A a \times A a$; progeny mated back to $A a$. This gives the same result as random mating; after $n$ inbreedings:

$$
A A={ }_{4}^{1} . \quad a a=\frac{1}{4} . \quad A a=\frac{1}{2} .
$$

(38 Parents $A A \times A a$; mated back to $A A$. After $n$ inbreedings:

$$
\begin{aligned}
A A & =\frac{\mathrm{C}_{n+1}}{\mathrm{~B}_{n+1}}=\frac{2^{n+1}-\mathrm{I}}{2^{n+1}} ; \frac{3}{4}, \frac{7}{8}, \frac{9}{16} \ldots \ldots \text { I } . A A_{6}=.992 . \\
a a & =0 . \\
A a & =\frac{\mathrm{I}}{2^{n+1}} ; \text { limit o. } A a=.008 .
\end{aligned}
$$

(39) Parents $A A$ and $A a$; progeny mated back to $A a$.

Here $A A$ gives the series $\mathrm{D}, a a$ gives the series $\mathrm{C}$, while $A a$ is always $1 / 2$. That is, after $n$ inbreedings:

$$
\begin{aligned}
A A & =\frac{\mathrm{D}_{n+1}}{\mathrm{~B}_{n+2}}\left(=\frac{2^{n}+\mathrm{I}}{2^{n+2}}\right) ; \frac{3}{8}, \frac{5}{16}, \frac{9}{32} \ldots \ldots \frac{1}{4} . \quad A A_{5}=.258 . \\
a a & =\frac{\mathrm{C}_{n}}{\mathrm{~B}_{n+2}}\left(=\frac{2^{n}-\mathrm{I}}{2^{n+2}}\right) ; \frac{1}{8}, \frac{3}{16}, \frac{7}{16} \ldots \ldots \frac{1}{4} . \quad a a_{5}=.242 . \\
A a & =\frac{1}{2} .
\end{aligned}
$$

(40) Parents $a a \times A a$; mated to $a a$. After $n$ inbreedings:

$$
a a=\frac{\mathrm{C}_{n+1}}{\mathrm{~B}_{n+1}} . \quad A a=\frac{\mathrm{I}}{\mathrm{B}_{n+1}} . \quad A A=0 .
$$

(4I) Parents $a a \times A a$; mated back to $A a$. After $n$ inbreedings:

$$
\begin{gathered}
a a=\frac{\mathrm{D}_{n+1}}{\mathrm{~B}_{n+2}}\left(=\frac{2^{n}+\mathrm{I}}{2^{n+2}}\right) ; \operatorname{limit} \frac{1}{4} . \quad A A=\frac{\mathrm{C}_{n}}{\mathrm{~B}_{n+2}}\left(=\frac{2^{n}-\mathrm{I}}{2^{n+2}}\right) ; \operatorname{limit} \frac{1}{4} . \\
A a=\frac{1}{2} .
\end{gathered}
$$

(42) In a Mendelian population consisting of $1 / 4 A A+1 / 4 a a+1 / 2$ $A a$, at a certain time the female progeny are bred back to their fathers, and this continues. Here $A A$ and $a a$ each gives the series $\mathrm{E}$, beginning at 5 , while $A a$ gives the series $\mathrm{D}$, beginning at 3 . That is, after $n$ inbreedings:

$$
\begin{aligned}
A A & =\frac{\mathrm{E}_{n+2}}{\mathrm{~B}_{n+3}} ; \frac{5}{16}, \frac{11}{3 \frac{1}{2}}, \frac{23}{84} \ldots \ldots .3 . A A_{4}=.367 . \\
a a & =A A . \\
A a & =\frac{\mathrm{D}_{n+1}}{\mathrm{~B}_{n+2}} ; \frac{3}{8}, \frac{5}{18}, \frac{9}{32} \ldots \ldots 1 . \quad A a_{5}=.258 .
\end{aligned}
$$


ii. Parent by offspring, alternating system

The system of continuous employment of a single individual as parent with successive generations of its own progeny could not be continued indefinitely, owing to the limited length of life of the individual. It is possible to conceive a system which could be continued indefinitely, by using each parent alternately as the parent of a second generation. That is, each individual would be employed twice, once for breeding with its own parent, once for breeding with its progeny. Such an alternating system is represented in figure 2. Such a system could not well be carried out with higher organisms, since it requires in alternate generations the mating back of the male children to their mother, and the mothers are of course less numerous than the male children. The system could, however, be carried out with certain plants. We shall deal with it without regard to practical considerations, for its theoretical interest. When in this system "parents" are bred to progeny, each "parent" plays (from a statistical or numerical point of view) the same part that the father plays in the continuous system.

(43) The original (unrelated) parents are $A A$ and $a a$, giving progeny that are all $A a$. These $A a$ are bred back to $A A$, and the progeny resulting from this are bred to their parent $A a$. Thus an alternating system is continued, as illustrated in figure 2.

After mating back to parents has thus occurred $n$ times:

$$
\begin{aligned}
A A & =\frac{\mathrm{I}_{n+1}}{\mathrm{~B}_{n+1}} ; \frac{2}{4}, \frac{3}{8}, \frac{8}{16} \ldots \ldots \frac{2}{3} . A A_{1 \overline{7}}=.657 . \\
a a & =\frac{\mathrm{H}_{n+1}}{\mathrm{~B}_{n+1}} ; 0, \frac{1}{8}, \frac{2}{16} \ldots \ldots \frac{1}{3} . \quad a a_{17}=.3235 . \\
A a & =\frac{\mathrm{F}_{n+1}}{\mathrm{~B}_{n}} ; \frac{1}{2}, \frac{2}{4}, \frac{3}{8} \ldots \ldots \circ . \quad A a_{21}=.008 .
\end{aligned}
$$

(44) The first cross is $a a$ by $A A$; the progeny are bred back to $a a$, and the alternating system continues. This gives the same result as (43), save that the proportions of $A A$ and $\alpha a$ must be interchanged.

(45) It may be observed that by the system given in (43) and (44), and illustrated in figure 2 , one of the original individuals ( $a$ in figure 2 ) plays a smaller part than all others, being employed as a parent but once, while others are employed twice. It is of interest to determine the results when all parents are equally represented. For this purpose, after the original parents $A . A$ and $A a$ have produced progeny $A a$, half of these progeny are mated to the parent $A A$, half to the parent $a a$. The resulting progeny are then mated to their younger parents $(A a)$, and the alternating system continued as before. Figure 3 illustrates this system. 
(In working out this system, the correct statistical results are obtained if in each generation we mate all the progeny first to one of the two parents, then to the other.)

In this case $A A$ and $a a$ each give the series J, while $A a$ gives (as in (43) and (44), the Fibonacci series F. That is, after $n$ inbreedings:

$$
\begin{aligned}
A A & =\frac{\mathrm{J}_{n}}{\mathrm{~B}_{n+1}} ; \frac{1}{4}, \frac{2}{8}, \frac{5}{16} \ldots \ldots \frac{1}{2} . A A_{17}=.490 . \\
a a & =A A . \\
A a & =\frac{\mathrm{F}_{n+1}}{\mathrm{~B}_{n}} ; \frac{1}{2}, \frac{2}{4}, \frac{3}{8} \ldots \ldots \circ . A a_{21}=.008 .
\end{aligned}
$$

(46) Original cross, $A A \times A a$; progeny bred back to $A A$, and thenceforth the alternating system of figure 2 is employed. Here $A A$ gives the series L, $a a$ gives the series $\mathrm{H}$, while $A a$ gives the Fibonacci series (F). After $n$ inbreedings:

$$
\begin{aligned}
& A A=\frac{\mathrm{L}_{n+2}}{\mathrm{~B}_{n+2}} ; \frac{6}{8}, \frac{11}{16}, \frac{24}{32}, \frac{48}{6}, \frac{99}{128} \ldots \ldots . .4 A_{14}=.824 . \\
& a \alpha=\frac{\mathrm{H}_{n+1}}{\mathrm{~B}_{n+2}} ; 0, \frac{1}{16}, \frac{2}{3^{2}}, \frac{6}{64}, \frac{13}{128} \ldots . \frac{1}{15} . \quad a a_{14}=.157 . \\
& A a=\frac{\mathrm{F}_{n+1}}{\mathrm{~B}_{n+1}} ; \frac{1}{4}, \frac{2}{8}, \frac{8}{16}, \frac{5}{32},{ }_{6}^{8}+\ldots .0 . A a_{17}=.0098 .
\end{aligned}
$$

(47) Original cross, $A A \times A a$; progeny bred back to $A a$, and thenceforth by the alternating system of figure 2. After $n$ such inbreedings:

$$
\begin{aligned}
A A & =\frac{\mathrm{I}_{n+2}}{\mathrm{~B}_{n+2}} ; \frac{3}{8}, \frac{8}{18}, \frac{16}{3} \ldots \ldots \frac{2}{3} . \quad A A_{16}=.657 . \\
a a & =\frac{\mathrm{H}_{n+2}}{\mathrm{~B}_{n+2}} ; \frac{1}{8}, \frac{2}{16}, \frac{6}{32} \ldots \ldots \frac{1}{3} . \quad a a_{16}=.323 . \\
A a & =\frac{\mathrm{F}_{n+2}}{\mathrm{~B}_{n+1}} ; \frac{2}{4}, \frac{3}{8}, \frac{5}{16} \ldots \ldots \circ . \quad A a_{20}=.008 .
\end{aligned}
$$

(48) If with parents $A A \times A a$ we make the part played by the two original parents equal, by following the scheme represented in figure 3 , we obtain after $n$ inbreedings the following results:

$$
\begin{aligned}
A A & =\frac{\mathrm{M}_{n+1}}{\mathrm{~B}_{n+3}} ; \frac{9}{16}, \frac{19}{82}, \frac{40}{8} \ldots \ldots .3 . A A_{15}=.740 \mathrm{I} . \\
a a & =\frac{\mathrm{K}_{n+1}}{\mathrm{~B}_{n+3}} ; \frac{1}{16}, \frac{3}{32}, \frac{8}{84} \ldots \ldots \frac{1}{4} . \quad a a_{15}=.240 \mathrm{I} . \\
A a & =\frac{\mathrm{F}_{n+3}}{\mathrm{~B}_{n+2}} ; \frac{3}{8}, \frac{5}{16}, \frac{8}{32} \ldots \ldots \circ . \quad A a_{19}=.008 .
\end{aligned}
$$

(49) Parents $a a \times A a$; alternating system of figure 2, beginning with aa. After $n$ inbreedings the proportions are as in (46), with those for $A A$ and $a a$ interchanged. That is: 


$$
a a=\frac{\mathrm{L}_{n+2}}{\mathrm{~B}_{n+2}} . \quad A A=\frac{\mathrm{H}_{n+1}}{\mathrm{~B}_{n+2}} . \quad A a=\frac{\mathrm{F}_{n+1}}{\mathrm{~B}_{n+1}} .
$$

(50) Parents $a a \times A a$; alternating system of figure 2 , beginning with $A a$. After $n$ such inbreedings the proportions are as in (47), with those for $A A$ and $a a$ interchanged.

(5I) Parents $a a \times A a$; equalized alternating system of figure 3 . After $n$ such inbreedings the proportions are as in (48), with those for $A A$ and $a a$ interchanged.

(52) Original parents $A a \times A a$. In this case the same results are reached whether we employ the system shown in figure 2 , or that shown in figure 3. After $n$ inbreedings:

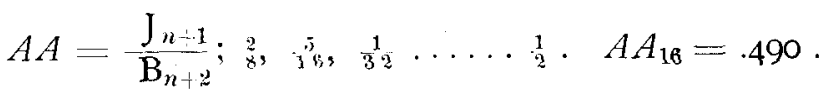

$$
\begin{aligned}
& a a=\frac{\mathrm{J}_{n+1}}{\dot{\mathrm{B}}_{\boldsymbol{n}+*}} .
\end{aligned}
$$

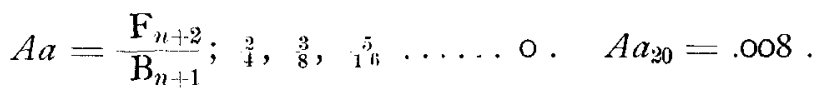

iii. Half the progeny bred to one parent, half to the other

It is theoretically interesting to examine what results would follow if half the progeny were bred each time to one of the parents, half to the other. This system could be carried out in plants in which vegetative reproduction by cuttings is possible. The daughters would be bred, as usual, to the father. From the mother-plant would be produced by cutting as many plants as there are sons, and one of these would be bred to each son. The relative numbers of progeny in the next generation would thus depend on the relative numbers of different kinds of their younger parents, as in the mating of father by daughters.

This system gives a number of peculiar series of results, seemingly not reducible to simple general formulae, so that I shall have to content myself with giving rules for obtaining any term of the series when preceding terms are known, together with a number of the earlier terms in each series.

(53) Original parents $A A \times a a$. Half the progeny are mated to one parent, half to the other, and this system is continued.

What we desire is to obtain the three series of fractions giving the proportions of $A A, a a$ and $A a$, for successive values of $n$. The results for $A a$ give the key to those for the others, so that $A a$ will be dealt with first. The rule obtained is such as to require that before its application the first two terms shall be known; these for $A a$ are: after I 
inbreeding $\frac{z}{4}$; after 2 inbreedings $\frac{6}{16}$. We clesire then to obtain a rule for finding the term in the series for any value of $n$, when the terms for $n-\mathrm{I}$ and $n-2$ are known.

To obtain the $n$th term of the series:

For $A a$ : If $n$ is odd, double the numerator and denominator of the term for $n-\mathrm{I}$, and from the resulting numerator subtract the numerator of the term for $n-2$. Thus, since the first term is $\frac{2}{4}$ and the second $\frac{6}{16}$, the third term is $12 \frac{2}{32}=\frac{2}{32}=\frac{10}{2}$.

If $n$ is even, multiply by four the numerator and denominator of the term for $n-\mathrm{I}$, and from the resulting numerator subtract the numerator of the term for $n-2$. Thus, as term 2 is $\frac{6}{16}$ and term 3 is $\frac{10}{32}$ term 4 will be $\frac{40}{1 \frac{2}{8}}=\frac{34}{128}$.

(The fractions expressing the values must not be reduced to lower terms, otherwise incorrect results are reached, or the numerators themselves become fractional.)

For $A A$ or $a a$ (the values are the same for both):

If $n$ is odd, double numerator and denominator of the term for $n-\mathrm{I}$, and to the resulting numerator add $1 / 2$ the numerator of $A a$ for $n-2$. Thus, term I is $1 / 4$; term 2 is $\frac{5}{16}$; therefore term 3 is $\frac{10+1}{32}=\frac{1}{3} \frac{1}{2}$ (since term I for $A a$ is $\frac{2}{4}$ ).

If $n$ is even, multiply by 4 the numerator and denominator of the term for $n-\mathrm{I}$, and add to the resulting numerator $1 / 2$ the numerator of $A a$ for $n-2$. Thus, since term 3 is $\frac{11}{32}$, term 4 will be $\frac{44+8}{128}=\frac{47}{128}$.

The first ten terms of the series are thus:

\begin{tabular}{lrrrrrrrrrr}
$r=$ & I & 2 & 3 & 4 & 5 & 6 & 7 & 8 & 9 & 10 \\
\hline$A a$ & 2 & 6 & I0 & 34 & 58 & 198 & 338 & II54 & 1970 & 6726 \\
$A A$ & I & 5 & II & 47 & 99 & 413 & 855 & 3519 & 7207 & 29405 \\
$a a$ & I & 5 & II & 47 & 99 & 413 & 855 & 3519 & 7207 & 29405 \\
Total & 4 & 16 & 32 & I28 & 256 & 1024 & 2048 & 8 I92 & 16384 & 65536
\end{tabular}

(It may be noted that five of these terms were worked out before the rule was obtained; that three more were then obtained through the rule and verified by working out in detail; while the last two are based on the rule alone.)

The limiting value for $A A$ and for $a a$ is apparently $1 / 2$; for $A a, 0$; but I have not carried the results far enough to make this certain.

(54) Original parents $A a \times A a$; half the progeny mated to one parent, half to the other.

The rules here are the same as in (53), save that the relations of odd and even are interchanged; also the series begin differently (that is, to 
obtain an odd-numbered term we multiply by 4 , etc., to get an evennumbered term we multiply by 2 , etc.). The series of proportions are:

\begin{tabular}{lrrrrrrrrrr}
$n=$ & $\mathbf{1}$ & 2 & 3 & 4 & 5 & 6 & 7 & 8 & 9 & 10 \\
\hline$A a$ & 8 & 14 & 48 & 82 & 280 & 478 & 1632 & 2786 & 9512 & 16238 \\
$A A$ & 4 & 9 & 40 & 87 & 372 & 785 & 3280 & 6799 & 28012 & 57417 \\
$a a$ & 4 & 9 & 40 & 87 & 372 & 785 & 3280 & 6799 & 28012 & 57417 \\
Total & 16 & 32 & 128 & 256 & 1024 & 2048 & 8192 & 16384 & 65536 & I31072
\end{tabular}

(55) Original parents $A A \times A a$; half the progeny mated regularly to each parent.

The rules here are the same as in (54), though the series begin differently and give diverse results. For $A a$, if $n$ is odd, multiply by 4 the numerator and denominator of term $n-\mathrm{I}$, and subtract numerator of $n-2$; if $n$ is even, multiply by 2 , and subtract numerator of $n-2$, etc. The first ten terms of the resulting series are:

\begin{tabular}{|c|c|c|c|c|c|c|c|c|c|c|}
\hline$n=$ & I & 2 & 3 & 4 & 5 & 6 & 7 & 8 & 9 & IO \\
\hline$\overline{A a}$ & 6 & Io & 34 & 58 & 198 & 338 & II 54 & 1970 & 6726 & $\overline{I I 482}$ \\
\hline$A A$ & 9 & I9 & 79 & 163 & 669 & I 367 & 5567 & I I 303 & 45789 & 92563 \\
\hline$a a$ & I & 3 & I5 & 35 & 157 & 343 & $147 \mathrm{I}$ & $3 \operatorname{III}$ & 13021 & 27027 \\
\hline ota & It & 32 & 128 & 256 & I024 & 2048 & 8192 & 16384 & 65536 & I3IC \\
\hline
\end{tabular}

It will be observed that here, as in $(3 \mathrm{r}),(A \dot{A}+\mathrm{I} / 2 A a)=3(a a+$ I/2 $A a$ ).

If the original parents were $a a$ and $A a$, doubtless the results would be the same, save with the proportions for $A A$ and $a a$ interchanged.

\section{SEX-LinkEd FACTORS}

The peculiarities of sex-linked factors are, for present purposes: (I) The males consist of but two classes with respect to such factors, while the females fall into the usual three classes. (2) The males are never double dominants; they never show the constitution $A A$. Two different methods of conceiving the constitution of the males are extant, resulting in two diverse methods of representing them. For our purposes both methods have the same results, but one is more convenient than the other. According to the absolute "presence and absence" theory, the two classes of males are to be designated $A a$ and $a a$; one is a heterozygote, the other a pure recessive, while no pure dominant exists. According to MoRGaN's results, the male is conceived to have but one factor, for either dominant or recessive, so that the two classes of males are to be represented as $A-$ and $a-$ Both systems of designation give (of course) in later generations the same proportions of the various classes of males and females. But the system which represents differ- 
ently the males and females is the more convenient in computing and representing results. We shall represent the two classes of males by $A-$ and $a-$, the three classes of females by $A A$, $a a$ and $A a$. Anyone who desires to do so may substitute $a$ for the dash in the formula for the males. The only difference produced will be to make it, in working out formulae, difficult to determine the proportions of males and females having any particular constitution, and as a consequence, to determine what later matings should be made.

The characteristic feature in the results with sex-linked characters is the frequent appearance of the series G, which is of such a nature (as will be seen) that the proportions of individuals having a given constitution do not change continuously in one direction, but show successive slight increases and decreases, though with a general trend in one direction.

In the formulae for sex-linked characters the proportions of the males and of the females will be given separately. In all cases the number of males and females is the same.

\section{a. Random mating}

(56) The population at the beginning consists of two biotypes in equal numbers, each with equal numbers of the two sexes; that is, on the one hand of $A A$ and $A-$, on the other of $a a$ and $a-$. These mate at random (any male with any female).

In any later generation the population is:

Males: $1 / 2 A-+1 / 2 a-$

Females: $1 / 4 A A+1 / 4 a a+1 / 2 A a$.

Of the total population $5 / 8$ are dominants, $3 / 8$ recessives.

(57) In the more general case the proportions at the beginning are: $r A A$ and $r A-; t a a$ and $t a-$ (there being thus two biotypes differing in number, but with equal numbers of males and females in each). After random mating for any number of generations the proportions are:

$$
\begin{aligned}
\text { Males : } A- & =\frac{r}{r+t} . \quad a-\cdots=\frac{t}{r+t} . \\
\text { Females : } A A & =\frac{r^{2}}{(r+t)^{2}} . \quad a a=\frac{t^{2}}{(r+t)^{2}} . \quad A a=\frac{2 r t}{(r+t)^{2}} .
\end{aligned}
$$

(58) The population at the beginning is $A A$ and $a-$ (two biotypes in equal numbers, but all the males belonging to one, all the females to the other). After the first cross all breeding is by random mating.

This case gives certain curious series of proportions as the number 
of successive random matings increases. The two sorts of males give the series $G$ (table $I$, page 56 ), while the three classes of females give series formed by products of succeeding terms of $G$, or compounds of these with B. That is, after $n$ generations of random mating (not including in $n$ the first cross) :

$$
\begin{aligned}
& \text { Males:- } \\
& A \longrightarrow=\frac{\mathrm{G}_{n+1}}{\mathrm{~B}_{n}} ; \frac{1}{2}, \frac{3}{4}, \frac{5}{8}, \frac{11}{16} \text {, etc. } \ldots \ldots \frac{2}{3} . A A_{6}=.672 . \\
& a-=\frac{\mathrm{G}_{n}}{\mathrm{~B}_{n}} ; \frac{1}{2}, \frac{1}{4}, \frac{3}{8}, \frac{5}{16}, \text { etc. } \ldots \ldots \frac{1}{3} . \quad a a_{6}=.328 .
\end{aligned}
$$

Females :-

$$
\begin{aligned}
& A A=\frac{\mathrm{G}_{n} \times \mathrm{G}_{n+1}}{\mathrm{~B}_{2 n-1}} ; \frac{1}{2}, \frac{3}{8}, \frac{15}{32}, \frac{55}{128}, \frac{281}{512}, \frac{903}{2048} \ldots \ldots \frac{4}{9} . A A_{5}=.45 \mathrm{I} . \\
& a a=\frac{\mathrm{G}_{n} \times \mathrm{G}_{n-1}}{\mathrm{~B}_{2 n-1}} ; 0, \frac{1}{8}, \frac{3}{32}, \frac{15}{128}, \frac{55}{512}, \frac{231}{2048} \ldots ._{9}^{1} . \quad a a_{5}=.107 . \\
& A a_{0}=\mathrm{I}-A A-a a ; \frac{1}{2}, \frac{4}{8}, \frac{14}{3}, \frac{58}{128}, \frac{226}{512}, \frac{914}{2048} \ldots \ldots \frac{4}{9} . \quad A a_{3}=.437 .
\end{aligned}
$$

The above series given by the different constitutions present some interesting points. Any term $n$ of the values for $A$ - is given by the sum of $n$ terms of the following well-known series:

$$
\frac{1}{2}+\frac{1}{4}-\frac{1}{8}+\frac{1}{10}-\frac{1}{32}+\ldots \text {. etc. }
$$

If we discontinue this series after any number $n$ of terms, and take its sum to that point, we shall have the proportion of $A-$ for $n$ random matings.

Similarly, the series of values for $a-$ are given by the sums of the following series, stopped at any point:

$$
\frac{1}{2}-\frac{1}{4}+\frac{1}{8}-\frac{1}{16}+\frac{1}{82}-\ldots \text {. etc. }
$$

In both cases the signs of the terms (after the first) alternate, and each successive denominator is double the preceding one.

In the females the values of the different terms are given by the sums of still more curious series. The nature of these series will best be understood by taking a concrete case. After 5 random matings $(n=5)$, the values for $A A, a a$ and $A a$ are given by the sums of the following series:

$$
\begin{aligned}
& a a_{5}=\frac{0}{2}+\frac{1}{4}-\frac{2}{8}+\frac{8}{16}-\frac{4}{32}+\frac{4}{84}-\frac{3}{128}+\frac{2}{256}-\frac{1}{512}=\frac{55}{512} . \\
& A A_{5}=\frac{1}{2}\left[+\frac{0}{4}-\frac{1}{8}+\frac{2}{16}-\frac{3}{32}+\frac{4}{84}-\frac{3}{128}+\frac{5}{256}-{ }_{51}^{1} \frac{1}{2}\right]=\frac{231}{512} . \\
& A a_{5}=\frac{1}{2}\left[-\frac{1}{4}+\frac{3}{8}-\frac{55}{16}+\frac{7}{82}-\frac{8}{84}+\frac{6}{128}-\frac{4}{256}+{ }_{5}^{\frac{2}{1}} \frac{1}{2}\right]=\frac{226}{512} \text {. }
\end{aligned}
$$

These series for any value of $n$ can be written by taking note of the following facts:

For aa: (I) The first term is $\frac{0}{2}$; (2) The total number of terms is 
$2 n-1$. (3) The terms after the first alternate in sign, the sign of the second term being + . (4) The numerators increase successively by $\mathrm{I}$ up to the number $n-\mathrm{I}$; the series of numerators is then repeated in reverse order, to I. (5) The denominator is doubled for each successive term.

For $A A$ : (I) The value is equal to $I / 2$, with the series within the brackets; (2) The series within the brackets begins with +4 ; (3) The number of terms in the brackets is $2 n-2$. (4) The terms of this series alternate in sign. (5) The numerators of its terms are increased successively by I, up to the number $n-\mathrm{I}$, then decrease by $\mathrm{I}$, to unity. (6) The denominator is doubled for each successive term.

For $A a$ : ( I) The value is $1 / 2$, with the series in brackets. (2) The first term of the series in brackets is $-1 / 4$. (3) The number of terms in the brackets is $2 n-2$. (4) The terms of this series alternate in sign. (5) The successive numerators are the successive odd numbers up to the number $(2 n-3)$, followed by the next higher even number, then by the descending series of even numbers, to 2 . (6) The denominator is doubled for each successive term.

(59) If the population at the beginning is $a a$ and $A$-, in equal numbers, the restlts are the same as in $(58)$, save that:

The values for $A$ - and $a-$ are to be interchanged;

The values for $A A$ and $a a$ are to be interchanged;

The values for $A a$ remain the same.

\section{b. Assortative mating}

(6o) The population consists of the progeny of $A A, a a, A-$ and $a$-, which were present in equal numbers and have mated at random. That is, the population consists (by (56)) of: females, $1 / 4 A A+$ $\mathrm{I} / 4 a a+\mathrm{I} / 2 A a ;$ males, $\mathrm{I} / 2 A-\mathrm{J} / 2 a_{1}-$. These thenceforth mate assortatively; the dominants $A A$ and $A a$ mate only with $A-$, while the recessive $a a$ mates only with $a$-.

In the case of sex-linked factors the number of dominant males is less than that of dominant females. It must be assumed that one male may fertilize several females, so that no proportion of any particular class of females goes unfertilized on account of relative scarcity of males. The proportions of progeny produced by dominants on the one hand, by recessives on the other, will therefore depend entirely on the relative proportions of dominant and recessive female parents (provided that, as is always the case, some considerable number of males of each 
class exist.) In working out the proportions in later generations, it must be assumed that all female dominants are fertilized by $A$-, all female recessives by $a-$. In this case $A-$ and $A A$ give the series E; $a$ - gives the series $\mathrm{D}$. After $n$ assortative matings, the proportions are:

$$
\text { Males: } \begin{aligned}
A- & =\frac{\mathrm{E}_{n+1}}{\mathrm{~B}_{n+1}} ; \frac{2}{4}, \frac{5}{8}, \frac{11}{16} \ldots \ldots \frac{3}{4} . \quad A-6=.742 . \\
a- & =\frac{\mathrm{D}_{n}}{\mathrm{~B}_{n+1}} ; \frac{2}{4}, \frac{3}{8}, \frac{5}{16} \ldots \ldots \frac{1}{4} . \quad a-_{6}=.258 .
\end{aligned}
$$

Females : $\quad A A=\frac{\mathrm{E}_{n+1}}{\mathrm{~B}_{n+1}} ; \frac{2}{4}, \frac{5}{8}, \frac{11}{16} \ldots . . \frac{3}{4} . \quad A A_{6}=.742$.

$$
\begin{aligned}
& a a=\frac{1}{4} . \\
& A a=\frac{\mathrm{I}}{\mathrm{B}_{n+1}} ; \frac{1}{4}, \frac{1}{8}, \frac{1}{16} \ldots \ldots \circ . \quad A a_{6}=.008 .
\end{aligned}
$$

(6I) At the beginning, $A A$ is crossed with $a$-, the progeny mate assortatively. The progeny are of course $A a+A-$, males and females in equal number.

In this method of mating it turns out that the recessives do not propagate at all, since no female recessives (aa) are formed, and the female heterozygotes of course mate with male dominants. If the females reproduce in proportion to the numbers of the two classes that exist ( $A A$ and $A a$ ), then after $n$ such assortative matings the population in the $n$th generation is:

Males: $A-=\frac{2^{n}-\mathbf{I}}{2^{n}} ; a-=\frac{\mathrm{I}}{2^{n}}$.

Females : $A A=\frac{2^{n}-\mathbf{I}}{2^{n}}$, limit I. $A A_{7}=.992$.

$$
A a=\frac{\mathrm{I}}{2^{n}} \text {; limit } 0 . \quad A a_{7}=008 .
$$

The same result is reached if we make at the beginning a reciprocal cross, $A A$ by $a$ - and $a a$ by $A$-, the progeny mating assortatively.

(62) The original cross is $a a$ by $A-$. This gives progeny $A a+$ $a$-, which gives no opportunity for assortative mating. If these are crossed again, they give $A a+a a+A-+a-$, in which assortative mating may occur. After $n$ successive assortative matings the population is:

$$
\begin{aligned}
\text { Males : } \quad A- & =\frac{\mathrm{C}_{n}}{\mathrm{~B}_{n+1}}\left(=\frac{2^{n}-\mathrm{I}}{2^{n+1}}\right) ; \frac{1}{4}, \frac{3}{8}, \frac{7}{16} \ldots \ldots \frac{1}{2} . \quad A-6=.492 . \\
a- & =\frac{\mathrm{D}_{n+1}}{\mathrm{~B}_{n+1}}\left(=\frac{2^{n}+1}{2^{n+1}}\right) ; \frac{3}{4}, \frac{5}{8}, \frac{9}{16} \ldots \ldots \frac{1}{2} . \quad a-6=.508 .
\end{aligned}
$$


Females: $\quad A A=\frac{\mathrm{C}_{n}}{\mathrm{~B}_{n+1}}\left(=\frac{2^{n}-\mathrm{I}}{2^{n+1}}\right)$.

$$
\begin{aligned}
& a a=\frac{1}{2} . \\
& A a=\frac{1}{2^{n+1}} .
\end{aligned}
$$

\section{c. Dominants alone selected}

If our original cross is either $A A$ by $a$-, or $a a$ by $A$ - (followed by crossing of their progeny $A a$ and $a-$ ) ; or we have the double reciprocal crosses $A A$ by $a-$ and $a a$ by $A$-; or it is $A a$ by $A$-; or $A a$ by $a$-, the same series of results is obtained after $n$ generations of breeding from dominants alone, but in some cases a given result is reached earlier than in others, thus requiring in the formulae a slightly different relation to $n$. I will therefore list the formulae for each case.

Original cross.

After $n$ selections of dominant alone:

(63) $A A$ by $a-$.

(64) aa by $A-$ (giving

$A a$ and $a-$, which

are bred together).

$\left.\begin{array}{l}\text { (65) Reciprocal crosses, } \\ A A \text { by } a-\text { and } a a \text { by } A-\end{array}\right)\left(\begin{array}{l}A a \text { or } a-\frac{1}{\mathrm{~B}_{n+1}}, \\ a a=0 \text {. }\end{array}\right.$

(66) $A a$ by $a-$

(67) $A a$ by $A-. \quad A A$ or $A-=\frac{\mathrm{C}_{n+1}}{\mathrm{~B}_{n+2}} \cdot A a$ or $a-=\frac{\mathrm{I}}{\mathrm{B}_{n+2}}$.

$$
a a=0 \text {. }
$$

(68) Original population $A A$, aa, $A$ - and $a-$, in equal numbers, mating at random so as to give: males $1 / 2 A-+1 / 2 a-;$ females $1 / 4$ $A A+1 / 4 a a+1 / 2 A a$. Now begins selection of dominants alone. After $n$ such selections:

$A A$ or $A-=\frac{\mathrm{E}_{n+1}}{3 \mathrm{~B}_{n-1}} ; \frac{2}{3}, \frac{5}{6}, \frac{11}{12}, \frac{23}{24} \ldots \ldots$ I . $A A_{7}$ or $A-7=.995$.

$A a$ or $a-=\frac{\mathrm{I}}{3 \mathrm{~B}_{n-1}} ; \frac{1}{3}, \frac{1}{8}, \frac{1}{1 \frac{1}{2}}, \frac{1}{24} \ldots \ldots$ o. $A a_{7}$ or $a-7=.005$ $a a=0$.

\section{INBREEDING AND SEX-LINKED FACTORS}

\section{d. Mating of brother by sister}

(69) Original cross, $A A \times a-$; brother and sister mating then continues for $n$ generations. 
Males : $A-=\frac{\mathrm{G}_{n+1}}{\mathrm{~B}_{n}} ; \frac{1}{2}, \frac{3}{4}, \frac{5}{8} \ldots . \frac{\frac{2}{3}}{3} . \quad A-_{6}=.672$.

$$
a-=\frac{\mathrm{G}_{n}}{\mathrm{~B}_{n}} ; \frac{1}{2}, \frac{1}{4}, \frac{3}{8} \ldots \ldots \frac{1}{3} \cdot a-6=.328 .
$$

Females : $A A=\frac{\mathrm{I}_{n+1}}{\mathrm{~B}_{n+1}} ; \frac{2}{4}, \frac{3}{8}, \frac{8}{16}, \frac{16}{32} \ldots \ldots \frac{2}{3} . \quad A A_{17}=.657$.

$$
\begin{aligned}
& a a=\frac{\mathrm{H}_{n+1}}{\mathrm{~B}_{n+1}} ; 0, \frac{1}{8}, \frac{2}{16}, \frac{6}{32} \ldots \ldots \frac{1}{3} . \quad a a_{17}=.324 . \\
& A a=\frac{\mathrm{F}_{n+1}}{\mathrm{~B}_{n}} ;{ }_{2}^{1}, \frac{2}{4}, \frac{3}{8}, \frac{5}{11} \ldots \ldots \circ . \quad A a_{21}=.008 .
\end{aligned}
$$

(70) The original cross is $a a$ by $A$ -

The results here are the same as in the last (69) save that:

The values for $A A$ and $a a$ are interchanged.

The values for $A$ - and $a-$ are interchanged.

The values for $A a$ are the same as in (69).

(71) At the beginning there are reciprocal crosses, $A A$ by $a$-, and aa by $A-$; thereupon brother by sister matings occur. After $n$ such matings, the population is:

Males : $A-=\frac{1}{2} . \quad a-=\frac{1}{2}$.

Females : $\quad A A=\frac{\mathrm{J}_{n}}{\mathrm{~B}_{n+1}}$; thus $\frac{1}{4}, \frac{2}{8}, \frac{5}{16} \ldots \ldots \frac{1}{2} . A A_{17}=.490+$.

$$
\begin{aligned}
a a & =\frac{\mathrm{J}_{n}}{\mathrm{~B}_{n+1}} . \\
A a & =\frac{\mathrm{F}_{n+1}}{\mathrm{~B}_{n}}=\frac{1}{2}, \frac{2}{4}, \frac{3}{8} \ldots \ldots \circ . A a_{21}=.008 .
\end{aligned}
$$

(72) The original cross is $A a$ by $A$ -

This gives the same result as (69), save that any given set of proportions is reached one generation sooner. That is, after $n$ inbreedings:

Males : $\quad A-=\frac{\mathrm{G}_{n+2}}{\mathrm{~B}_{n+1}} . \quad a-=\frac{\mathrm{G}_{n+1}}{\mathrm{~B}_{n+1}}$.

Females : $\quad A A=\frac{\mathrm{I}_{n+2}}{\mathrm{~B}_{n+2}} . \quad a a=\frac{\mathrm{H}_{n+2}}{\mathrm{~B}_{n+2}} . \quad A a=\frac{\mathrm{F}_{n+2}}{\mathrm{~B}_{n+1}}$.

(73) The original cross is $A a$ by $a$ -

Results as in (72) save that:

The proportions for $A-$ and $a-$ are interchanged.

The proportions for $A A$ and $a a$ are interchanged.

The values for $A a$ are the same. 


\section{e. Mating of parent by offspring}

i. Same parent (father) used successively

(74) The parents are $A$ - and $a a$; after this cross the female progeny are bred back to their father, this continuing for $n$ generations.

Of the males:

$$
\begin{aligned}
A-=\frac{\mathrm{C}_{n}}{\mathrm{~B}_{n}}\left(\text { or } \frac{2^{n}-1}{2^{n}}\right) ; \text { limit I. } A-7=.992 . \\
\qquad-\frac{\mathrm{I}}{\mathrm{B}_{n}} \text {; limit 0. } a-7=.008 .
\end{aligned}
$$

Of the females:

$$
A A=\frac{\mathrm{C}_{n}}{\mathrm{~B}_{n}}\left(\text { or } \frac{2^{n}-\mathrm{I}}{2^{n}}\right) . \quad a a=0 . \quad A a=\frac{\mathrm{I}}{\mathrm{B}_{n}} .
$$

(75) The parents are $A-$ and $A a$; the female progeny are bred to their father for $n$ successive generations.

Of the males:

$A-=\frac{\mathrm{C}_{n+1}}{\mathrm{~B}_{n+1}} ;$ limit I. $A--_{6}=.992 . \quad a-=\frac{\mathrm{I}}{\mathrm{B}_{n+1}} ;$ limit $0 . \quad a-_{6}=.008$.

Of the females:

$$
A A=\frac{\mathrm{C}_{n+1}}{\mathrm{~B}_{n+1}},\left(\text { or } \frac{2^{n+1}-\mathrm{I}}{2^{n+1}}\right) . \quad a a=0 . \quad A a=\frac{\mathrm{I}}{\mathrm{B}_{n+1}} .
$$

(76) The parents are $a$ - and $A A$; after the cross the female progeny are bred to their father.

This gives the same result as (74), save that the results for $A$ - are to be interchanged with those for $a-$; also the results for $A A$ with those for $a a$, while $A a$ is unaltered.

(77) Parents $a-$ and $A a$; successive female progeny bred to father.

Results as in (75), with $A$ - and $a$ - interchanged; also $A A$ and $a a$ are interchanged.

ii. All female progeny mated to father, all male progeny to mother

It may be of theoretical interest to examine further what would be the result with sex-linked characters if all the daughters were mated to their father, all the sons to their mother. Such a system could be carried out with bisexual plants that could be propagated by vegetative cuttings. The mating of the daughters with the father would present no difficulty. For the mating of sons with their mother, cuttings would be taken of the mother plant, in number equal to the sons, and each son mated to one of these. 
(78) Original cross $A A$ by $a-$; daughters thereafter mated to their fathers, sons to their mothers.

Here in the case of the males, $A$ - gives the odd terms of the series $\mathrm{G}$, beginning with $\mathrm{G}_{3}=3$, while $a-$ gives the even terms of this series, beginning with $G_{2}=I$. The females give other modifications of this same series. After $n$ inbreedings:

Males :

$$
\begin{aligned}
& A-=\frac{\mathrm{G}_{2 n+1}}{\mathrm{~B}_{2 n}} ; \stackrel{3}{4}, \frac{11}{16}, \frac{43}{64}, \frac{1}{2} \frac{1}{5} \ldots \ldots \frac{2}{3} . \quad A-3=.672 . \\
& a-=\frac{\mathrm{G}_{2 n}}{\mathrm{~B}_{2 n}} ; \frac{1}{4}, \frac{3}{16}, \frac{21}{64}, \frac{8}{25^{5}} \ldots \ldots \frac{2}{3} . \quad a-3=.328 .
\end{aligned}
$$

Females :

$$
\begin{aligned}
& A A=\frac{\mathrm{G}_{2 n+2}-3^{n-1}}{\mathrm{~B}_{2 n+1}} ; \frac{4}{8}, \frac{18}{3 \frac{8}{2}}, \frac{76}{12}, \frac{314}{51 \frac{1}{2}} \ldots \ldots \frac{2}{3} . \quad A A_{10}=.657 . \\
& a a=\frac{\mathrm{G}_{2 n+1}-3^{n-1}}{\mathrm{~B}_{2 n+1}} ; \frac{2}{8}, \frac{8}{32}, \frac{34}{128}, \frac{144}{512} \ldots \frac{1}{3}_{3} . \quad a a_{10}=.324 . \\
& A a=\frac{3^{n-1}}{\mathrm{~B}_{2 n}} ; \frac{1}{1},{ }_{16}^{3}, \frac{9}{97}, \frac{25}{25} \ldots \ldots \circ . \quad A a_{13}=.008 .
\end{aligned}
$$

(79) The parents are $a a$ and $A-$; breeding as in (78).

Results as in (78), save that the proportions for $A-$ and $a-$ must be interchanged; also the proportions for $A A$ and $a a$ must be interchanged. Aa remains the same.

(80) At the beginning we make the reciprocal crosses $A A$ by $a-$ and $a a$ by $A-$; thereafter daughters are mated to fathers, sons to mothers. After $n$ such matings:

Males: $\quad A-=\frac{1}{2} . \quad a-=\frac{1}{2}$.



$$
\begin{aligned}
& \alpha q=A A \text {. }
\end{aligned}
$$

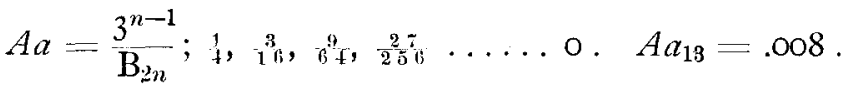

(8I) Original parents $A a$ by $A$-; thereafter daughters are mated to fathers, sons to mothers. After $n$ such matings:

Males :

$$
\begin{array}{ll}
A-=\frac{\mathrm{G}_{2 n+2}}{\mathrm{~B}_{2 n+1}} ; \frac{5}{8}, \frac{21}{3}, \frac{85}{128}, \frac{341}{512} \ldots \ldots \frac{2}{3} . & A-3=.664 . \\
a-=\frac{\mathrm{G}_{2 n+1}}{\mathrm{~B}_{2 n+1}} ; \frac{3}{8}, \frac{11}{32}, \frac{43}{128}, \frac{1}{517 \frac{1}{2}} \ldots \ldots \frac{1}{3} . \quad a-3=.336 .
\end{array}
$$


Females :

$$
\begin{aligned}
& A A=\frac{\mathrm{G}_{2 n+3}-3^{n}}{\mathrm{~B}_{2 n+2}} ;{ }_{1}^{8} \overline{5}, 3 \frac{4}{4}, \frac{14}{25},{ }_{10}^{602} \ldots \ldots \frac{2}{3} . \quad A A_{12}=.659 . \\
& a a=\frac{\mathrm{G}_{2 n+2}-3^{n}}{\mathrm{~B}_{2 n+2}} ; 2_{16}^{2}, \frac{12}{64}, \frac{58}{256}, \frac{260}{1^{2}} \ldots \ldots \frac{1}{8} . \quad a a_{12}=.326 . \\
& A a=\frac{3^{n}}{\mathbf{B}_{2 n-1}} ; \frac{3}{8}, \frac{9}{32}, \quad \frac{27}{1^{2} 8}, \frac{81}{512} \ldots \ldots \text { o. } \quad A a_{14}=.009 .
\end{aligned}
$$

(82) Parents $A a$ by $a-$; breeding as in ( $8 \mathrm{I}$ ).

Results as in $(8 \mathrm{r})$, but the proportions of $A$ - and $a-$ must be interchanged, as must also those for $A A$ and $a a$, while $A a$ remains unaltered.

The results when two or more factors, independent or linked, are considered, will be dealt with in a later paper.

\section{SUMMARY}

This paper gives formulae for finding in any generation the results of continued breeding by a given system, with respect to a single pair of alternative characters. Sex-linked characters and typical characters are dealt with separately. Formulae are given for the results of: random mating; assortative mating; selection of dominants; self-fertilization; inbreeding of brother by sister and of parent by offspring (several systems). In each case the diverse results obtained by beginning with different parental combinations are given.

It is shown that the results in successive generations form fractions such as may be obtained by compounding in various ways several well known arithmetical series. The first twenty terms are given of fourteen such series; the formulae show how the terms of these series are to be compounded in order to give the results of a particular type of breeding for any designated number of generations.

In addition to the general formulae, there are given the first three terms of each series of fractions obtained by given methods of breeding, and the limiting value toward which the series tend. These limiting values show the approximate proportion of the population that will have a given constitution after breeding by a given system for an indefinitely large number of generations. Furthermore, the paper gives the least number of generations of breeding by a given system required to approach within one percent of the final limit. This number is usually relatively small; after it is passed, continued breeding by the same system has little effect in changing the composition of the population. 
The results of the combinations given by diverse original parents and the several diverse systems of breeding require for their presentation eighty-two numbered formulae.

August 25. I915.

\section{LITERATURE CITED}

FISH, H. D., IgI4 On the progressive increase of homozygosis in brother-sister matings, Amer. Nat. 48: $759-76 \mathrm{I}$.

Hardy, G. H., 1908 Mendelian proportions in a mixed population. Science, 28: 49-50.

Jennings, H. S., I9I2 The production of pure homozygotic organisms from heterozygotes by self-fertilization. Amer. Nat., $45: 487-49$ r.

I9.4 Formulae for the results of inbreeding. Amer. Nat., 48: 693-696.

PEARL, R., 19I3 A contribution towards an analysis of the problem of inbreeding. Amer. Nat., 47: 577-6I.4.

I9I4 a On the results of inbreeding a Mendelian population; a correction and extension of previous conclusions. Amer. Nat., 48: $57-62$.

I9:4 b On a general formula for the constitution of the $n^{\prime}$ th generation of a Mendelian population in which all mating are of brother $X$ sister. Amer. Nat., $48: 49 \mathrm{I}-494$.

PeArsox, K., I904 On a generalized theory of alternative inheritance, with special reference to Mendel's laws. Phil. Trans. Roy. Soc. (A), $203: 53-86$. 